\title{
Über die Datenakzessivität in Printwörterbüchern. Einblicke in neuere Entwicklungen einer Theorie der Wörterbuchform
}

\author{
Herbert Ernst Wiegand, Germanistisches Seminar, Universität Heidelberg, \\ Heidelberg, Bundesrepublik Deutschland \\ (herbert.ernst.wiegand@gs.uni-heidelberg.de)
}

Zusammenfassung: In diesem Beitrag werden die Begriffe eingeführt, die man benötigt, um das Datenakzessivitätsprofil von Printwörterbüchern genau beschreiben zu können. Es wird zwischen externer und interner Datenakzessivität unterschieden; erstere ist obligatorisch, letztere ist fakultativ. Die externe Datenakzessivität wird durch äußere, die interne Datenakzessivität durch innere Zugriffsstrukturen innerhalb von akzessiven Wörterbucheinträgen hergestellt. Es werden unterschiedliche Typen von äußeren Zugriffsstrukturen als lineare Ordnungsstrukturen, wie z.B. alphabetische äußere Zugriffsstrukturen, numerische mediostrukturelle Zugriffsstrukturen, Registerzugriffsstrukturen u.a., beschrieben und ihr Funktionieren erklärt. Weiterhin werden äußere Zugriffsstrukturen von Zugriffspfaden abgegrenzt, die durch die Ausführung externer Zugriffshandlungen der Benutzer etabliert werden. Der Beitrag gibt insgesamt fünf Einblicke, aber keine zusammenhängende Übersicht über die vielfachen Ausprägungen der Eigenschaften der Wörterbuchform, die die Datenakzessivität sicherstellen.

Stichwörter: AKZESSIVER WÖRTERBUCHEINTRAG, ALPHABET, ALPHABETISCHE ÄUBERE ZUGRIFFSSTRUKTUR, ÄUBERER ZUGRIFFSPFAD, EXTERNE DATENAKZESSIVITÄT, EXTERNE ZUGRIFFSHANDLUNG, INNERE ZUGRIFFSSTRUKTUR, INTERNE DATENAKZESSIVITÄT, MEDIOSTRUKTURELLES LEITELEMENT, MEDIOSTRUKTURELLE ZUGRIFFSSTRUKTUR, MONOAKZESSIVES WÖRTERBUCH, REGISTER, REGISTERZUGRIFFSSTRUKTUR, POLYAKZESSIVES WÖRTERBUCH, SCHNELLZUGRIFFSSTRUKTUR, ZUGRIFFSTEXTELEMENT

\begin{abstract}
On the Data Accessibility in Printed Dictionaries. Insights into Recent Developments of a Theory of the Form of Dictionaries. In this contribution, concepts are introduced that are needed for a precise description of the data accessibility profile of printed dictionaries. A distinction is made between external and internal data accessibility, with the first being obligatory and the second being optional. The external data accessibility is presented by means of external access structures and the internal data accessibility by means of internal access structures in accessible dictionary entries. Distinctive types of external access structures as linear ordering structures, e.g. alphabetical external access structures, numerical mediostructural

* Dieser Beitrag basiert auf zwei unterschiedlich gestalteten Vorträgen, die ich im Juni 2005 an den Universitäten Erlangen und Essen gehalten habe.
\end{abstract}


access structures and register access structures are described and their functions explained. External access structures are furthermore distinguished from access routes, established by the user by means of the execution of external access procedures. The contribution gives a total of five insights but no coherent overview of the multiple manifestations of the characteristics of the form of dictionaries established by the data accessibility.

Keywords: ACCESS TEXTUAL ELEMENT, ACCESSIBLE DICTIONARY ENTRY, ALPHABET, ALPHABETICAL EXTERNAL ACCESS STRUCTURE, EXTERNAL ACCESS PROCEDURE, EXTERNAL ACCESS ROUTE, EXTERNAL DATA ACCESSIBILITY, INTERNAL ACCESS STRUCTURE, INTERNAL DATA ACCESSIBILITY, MEDIOSTRUCTURAL ACCESS STRUCTURE, MEDIOSTRUCTURAL GUIDING ELEMENT, MONO-ACCESSIBLE DICTIONARY, POLYACCESSIBLE DICTIONARY, RAPID ACCESS STRUCTURE, REGISTER, REGISTER ACCESS STRUCTURE

\section{Vorbemerkung}

Printwörterbücher gehören zur wissensvermittelnden Literatur. Dies ist nicht so $\mathrm{zu}$ verstehen, dass Wissen in den Wörterbüchern $\mathrm{zu}$ finden ist, sondern vielmehr so, dass anhand der lexikographischen Daten Wissen von den Benutzern erarbeitet werden kann. Wissen ist ein kognitives Phänomen von Personen, das u.a. anhand von semiotisch gebundenem Wissen und damit vor allem anhand von geäußerten Sprachzeichen erhältlich ist. ${ }^{1}$ Innerhalb der wissensvermittelnden Literatur haben die Wörterbücher eine Sonderstellung: Sie sind vor allem — wenn auch nicht nur — zum Nachschlagen konzipiert; entsprechend gilt einerseits: Ein gedrucktes Buch, in dem man nicht nachschlagen kann, wird nicht als Printwörterbuch betrachtet. Es ist aber andererseits nicht jedes Buch, das zur wissensvermittelnden Literatur gehört und in dem man beispielsweise anhand eines Namenregisters und/oder eines Sachregisters und/oder eines Wortregisters Daten nachschlagen kann, ein Wörterbuch. Die externe Datenakzessivität als eine Eigenschaft der Wörterbuchform, oder anders ausgedrückt - die Nachschlagbarkeit und damit die Zugriffsbereitschaft der lexikographischen Daten für einen Zugriff, der von außen auf ein Wörterbuch erfolgt, ist für die Frage, ob ein gedrucktes Werk als Wörterbuch gelten kann, nur eine objektiv notwendige und keine objektiv hinreichende Bedingung. Der Frage, welche Eigenschaften der Wörterbuchform und/oder des Wörterbuchgegenstandes (sensu Wiegand 1998: 302) als objektiv hinreichende Bedingung zu gelten haben, werde ich hier nicht nachgehen, weil dann ein ganz anderes Thema zu behandeln wäre.

Gerade habe ich festgestellt, dass Wörterbücher vor allem zum Nachschlagen konzipiert sind. Dies möchte ich so verstanden wissen: Es gibt einerseits Wörterbücher, die ausschließlich zum Nachschlagen in dem Sinne konzipiert sind, dass ein Zugriff auf die Daten von außerhalb des Wörterbuchs kommend erfolgen kann; andererseits gibt es Wörterbücher, die nicht ausschließlich für 
das Nachschlagen von außen und damit für den direkten externen Zugriff auf Daten anhand von Leitelementen konzipiert sind, sondern auch zum wörterbuchinternen Recherchieren mittels eines Systems von Verweisungen zum Zwecke der Vermittlung von Wissenszusammenhängen, die durch die wörterbuchinterne Datendistribution auf zugriffsverschiedene Positionen nicht erkennbar sind. In den erstgenannten Wörterbüchern ist die Organisation der Datenakzessivität weniger komplex als in den Letztgenannten.

Ich gehe daher in diesem einführenden Beitrag wie folgt exemplarisch vor: Ich führe zunächst die für das Thema benötigten grundlegenden lexikographietheoretischen Termini am Beispiel von Wörterbüchern ein, die ausschließlich zum Nachschlagen konzipiert sind. Dann gehe ich zu solchen Wörterbüchern über, bei denen die Datenakzessivität aufgrund der Existenz von Verweisungen und integrierten Umtexten komplexer ist. ${ }^{2}$ Insgesamt gebe ich fünf Einblicke, die aufeinander abgestimmt sind.

\section{Akzessive Wörterbucheinträge, externe versus interne Datenakzessi- vität}

Für die Datenakzessivität von gedruckten Nachschlagewerken jeder Art, also auch für die Datenakzessivität von nichtlexikographischen Nachschlagewerken, gilt das Folgende: Man muss zwischen der externen Datenakzessivität und der internen Datenakzessivität unterscheiden. Die externe Datenakzessivität wird durch die Einrichtung von äußeren Zugriffsstrukturen erreicht. Äußere Zugriffsstrukturen sind diejenigen Komponenten der Wörterbuchform, die sicherstellen, dass ein Wörterbuch akzessive Wörterbucheinträge und damit akzessive Daten aufweist. ${ }^{3}$ Akzessive Wörterbucheinträge, die im Englischen basic reference units heißen, sind die Grundbausteine jedes Nachschlagewerkes; die Wörterbuchartikel sind die wichtigsten. Ein Ausschnitt aus einer Typologie von akzessiven Wörterbucheinträgen findet sich in Abb. 1. Um bereits hier einem möglichen Irrtum vorzubeugen, sei festgestellt: Nicht alle äußeren Zugriffsstrukturen sind solche, auf die man von außerhalb eines Wörterbuchs zugreifen kann. Das Kriterium dafür, dass eine Zugriffsstruktur eine äußere Zugriffsstruktur ist, besteht mithin nicht darin, dass man von außerhalb des Wörterbuchs kommend zugreifen kann.

Die interne Datenakzessivität wird durch die Einrichtung von inneren Zugriffsstrukturen erreicht. Innere Zugriffsstrukturen dienen dem kundigen Benutzer (sensu Wiegand 1998: 506) zur Orientierung innerhalb akzessiver Wörterbucheinträge, insonderheit innerhalb von kondensierten Wörterbuchartikeln. Äußere Zugriffsstrukturen sind für gedruckte Nachschlagewerke obligatorisch; innere Zugriffsstrukturen sind dagegen fakultativ. Äußere und nichtalphabetische innere Zugriffsstrukturen unterscheiden sich — wie wir noch genauer im 4 . Abschnitt sehen werden - in ihren Zugriffseigenschaften grundsätzlich. 


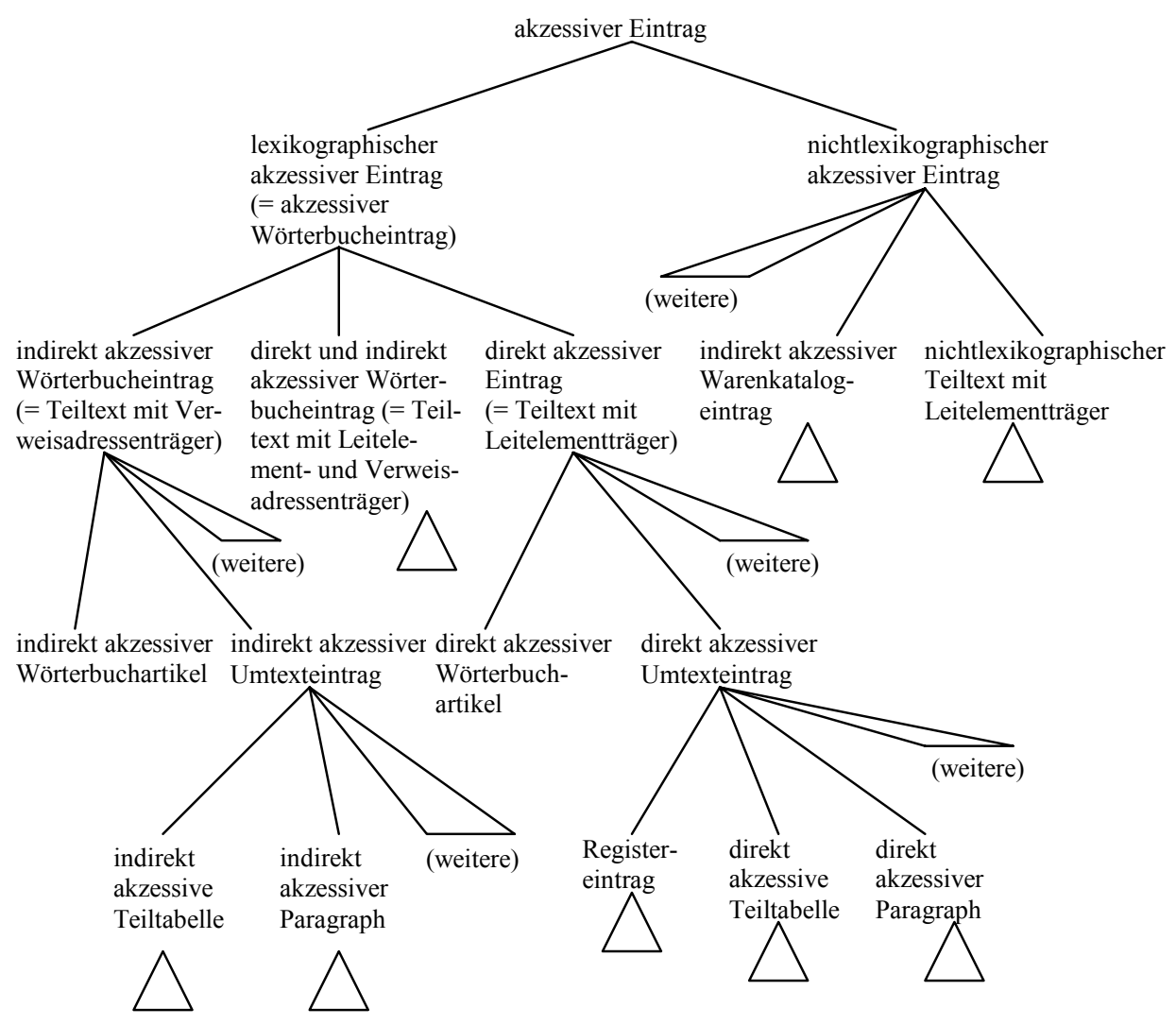

Abb. 1: Ausschnitt aus einer Typologie akzessiver Einträge; vgl. Wiegand 2003: 196.

\section{Externe Datenakzessivität}

Wir betrachten zunächst die externe Datenakzessivität für den einfachsten Fall, und zwar bei gedruckten Sprachwörterbüchern, deren Wörterbuchgegenstandsbereich (sensu Wiegand 1998: 303) solche Sprachen sind, die nach einem Alphabet verschriftet sind. Als Beispiel für einen Wörterbuchgegenstandsbereich nehmen wir die deutsche Standardsprache der Gegenwart. Zu deren Schriftsystem gehört das lateinische Alphabet in einer bestimmten Ausprägung: Es weist 26 Buchstaben auf. Unter dem Gesichtspunkt der Datenakzessivität ist dann der einfachste Fall ein glattalphabetischer monoakzessiver Einbänder der deutschen Standardsprache, der ausschließlich zum Nachschlagen von außen konzipiert ist, so dass es in ihm auch keine Verweisungen gibt. ${ }^{4}$ 


\subsection{Erster Einblick: Alphabet und alphabetische äußere Zugriffsstruktu- ren}

Will man verstehen, was eine alphabetische äußere Zugriffsstruktur ist, muss man als Verstehensvoraussetzung einige Eigenschaften des Alphabets kennen. Hier ist zunächst auf einen durchaus störenden unterschiedlichen Gebrauch der Wörter alphabetisch und Alphabet in verschiedenen wissenschaftlichen Disziplinen kurz einzugehen. In der Theorie der formalen Sprachen wird mit Alphabet eine nichtleere Menge von Zeichen bezeichnet, die nicht geordnet sein muss (vgl. Wiegand 1989: 374). Dies bedeutet: Die wohlgeformten Ketten über diesem Alphabet können nicht alphabetfundiert geordnet werden. Dieser Gebrauch von Alphabet ist für die Lexikographie ungeeignet. In der Schriftlinguistik ist der Gebrauch unterschiedlich. Die meisten Autoren verstehen unter einem Alphabet eine endliche Menge von Schriftzeichen, die eine historisch entstandene kanonische Abfolge aufweisen, so dass ein Alphabet eine endliche geordnete Menge ist. Diese Auffassung ist für die Lexikographie und Wörterbuchforschung dann brauchbar, wenn man sie präzisiert. Relativ zu diesem schriftlinguistischen Gebrauch heißt alphabetisch dann, dass eine bestimmte endliche Menge von sprachlichen Ausdrücken gemäß einem Alphabet geordnet ist. Wir werden noch genauer sehen, wie ungenau eine solche Redeweise ist. Als Hinweis zu dieser Feststellung an dieser Stelle nur dies: Man versuche einmal die deutschen Wörter Märchen, Öl, müde und heiß nach dem lat. Alphabet $\mathrm{zu}$ ordnen. Andere Autoren sprechen immer dann von der Alphabetreihe, wenn es nicht um den Verschriftungsaspekt, sondern um den Ordnungsaspekt eines Alphabets geht. ${ }^{5}$ Obwohl gerade dieser Terminus auf einen für die Lexikographie wesentlichen Aspekt jedes Alphabets aufmerksam macht, den wir noch genauer kennen lernen werden, werde ich beim Terminus Alphabet bleiben. In der lexikographischen Werkstattsprache wird Alphabet jargonhaft verwendet; werkstattsprachlich kann man z.B. formulieren: „Einige Ausdrücke sind falsch ins Alphabet eingeordnet." Gegen solche handlungsbegleitenden und/oder handlungskommentierenden werkstattsprachlichen Redewesen ist nichts einzuwenden, wenn sie bei den Beteiligten verständlich sind. In Umtexten von Wörterbüchern ist dieser Gebrauch von Alphabet jedoch fehl am Platz, auch wenn er sich in den Metatexten deutscher Wörterbücher öfters findet; statt vieler gebe ich nur ein Beispiel. In Mackensen (1977: V) heißt es: „Die geographischen Benennungen wurden in das Alphabet ebenso eingearbeitet wie die [...] Abbildungen." In allen lexikographietheoretischen Zusammenhängen ist dieser oder jeder andere werkstattsprachliche Gebrauch von Alphabet selbstverständlich unangemessen.

Im Folgenden gehe ich kurz auf Alphabete am Beispiel des lateinischen Alphabets des neuhochdeutschen Schriftsystems ein. Jedes Alphabet lässt sich unter zwei Aspekten betrachten, nämlich dem Verschriftungs- und dem Ordnungsaspekt. Anders ausgedrückt heißt das: Jedes Alphabet ist bifunktional: Es dient der Verschriftung einer gesprochenen Sprache und hat damit einen Bezug zu Lauten und Phonemen. Weiterhin ist es ein Mittel zur Herstellung von Ord- 
nung(en). Welcher der beiden Aspekte historisch der ältere ist, ist unter den "Schriftgelehrten“ strittig und interessiert hier nicht. Klar ist allerdings Folgendes: Die Universalität des Alphabets besteht darin, dass es als Mittel zur Herstellung von Ordnungen gerade dann am besten funktioniert, wenn man von jedem Laut-Buchstaben-Bezug vollständig abstrahiert. Für den Ordnungsaspekt des lat. Alphabets ist vielmehr nur von Relevanz, dass es sich um eine Ordnungsstruktur im mathematischen Sinne handelt, welche die Axiome einer totalen (oder: linearen) Ordnung erfüllt, so dass Isomorphien zur Zahlenreihe definiert werden können. Dies ist — sehr vereinfacht und ausdrücklich für Leser ohne mathematische Vorkenntnisse dargestellt — folgendermaßen zu verstehen:

Betrachten wir einmal ein lat. Alphabet in seiner üblichen Schreibweise im Vergleich mit den ersten 26 Zahlen der Zahlenreihe:

$$
\begin{aligned}
& 1<2<3<4<5<6<7 \\
& \begin{array}{lllllll}
a & b & c & d & e & f & g
\end{array} \\
& 8<9<10<11<12<13<14 \\
& \text { h } \begin{array}{llllll}
\mathrm{i} & \mathrm{j} & \mathrm{k} & \mathbf{1} & \mathbf{m} & \mathbf{n}
\end{array} \\
& 15<16<17<18<19<20<21 \\
& \begin{array}{lllllll}
\mathbf{0} & \mathbf{p} & \mathbf{q} & \mathbf{r} & \mathbf{s} & \mathbf{t} & \mathbf{u}
\end{array} \\
& 22<23<24<25<26 \\
& \begin{array}{lllll}
\mathbf{v} & \mathbf{w} & \mathbf{x} & \mathbf{y} & \mathbf{z}
\end{array}
\end{aligned}
$$

Dann kann man z.B. das Folgende wahrnehmen, nämlich direkt sehen (!!):

a geht $\mathrm{b}$ voraus; dem entspricht $1<2$ (zu lesen wie 1 ist kleiner als 2$)$,

a geht $\mathrm{c}$ voraus; dem entspricht $1<3$,

a geht $\mathrm{d}$ voraus; dem entspricht $1<4$,

a geht e voraus; dem entspricht $1<5$ usw. bis

a geht $\mathrm{z}$ voraus, was $1<26$ entspricht.

Oder man sieht z.B.: b geht $\mathrm{c}$ voraus, $\mathrm{c}$ geht $\mathrm{d}$ voraus usw. sowie die dazugehörigen Entsprechungen in der Zahlenreihe.

Das Alphabet in seiner kanonischen Schreibweise ist daher nichts weiter als eine geniale verkürzte, auf die Anschauung rekurrierende Präsentation einer Menge von 26 Buchstaben, auf der eine zweistellige Ordnungsrelation vom Typ der Vorgänger-Nachfolger-Relation (oder: der Präzedenzrelation) definiert ist, zu welcher der Relationsterm $x$ geht $y$ voraus oder der als synonym geltende Relationsterm $x$ vor $y$ gehört. Eine solche Relation lässt sich (in extensionaler Schreibweise) auch als Menge $M$ von geordneten Paaren hinschreiben; sehr stark abgekürzt wäre die Schreibweise wie folgt:

$$
\mathrm{M}=\{\langle\mathrm{a}, \mathrm{b}\rangle,\langle\mathrm{a}, \mathrm{c}\rangle,\langle\mathrm{a}, \mathrm{d}\rangle,\langle\mathrm{a}, \mathrm{e}\rangle,\langle\mathrm{a}, \mathrm{f}\rangle, \ldots,\langle\mathrm{y}, \mathrm{z}\rangle\} .
$$


Bei einer vollständigen Präsentation in dieser Mengenschreibweise müssten in der Schweifklammer genau $n \cdot(n-1): 2$ geordnete Paare stehen, mit $n=26$, also $(26 \cdot 25): 2=325$ geordnete Paare. Für alle 325 geordnete Paare der Relation geht der Relationsterm $x$ geht $y$ voraus immer dann in einen wahren Satz über, wenn für " $\mathrm{x}^{\prime \prime}$ die erste und für " $\mathrm{y}$ " die zweite Komponente eines geordneten Paares eingesetzt wird. Für das Paar $(a, b)$ ergibt sich dann: $a$ geht $b$ voraus. Oder für das Paar $(b, z)$ ergibt sich: $b$ geht $z$ voraus.

Eine auf einer Menge $M$ definierte zweistellige Ordnungsrelation $R_{p}$, die eine Teilmenge des kartesischen Produkts $M \times M$ ist (also $R_{p} \subseteq M \times M$ ) vom Typ der Präzedenzrelation hat folgende Eigenschaften, die als Ordnungsaxiome fungieren: $R_{p}$ ist (1) transitiv, (2) asymmetrisch und (3) konnex.

Für die Präzedenzrelation, die auf der Menge der 26 Buchstaben des lat. Alphabets definiert ist, kann dies informell folgendermaßen erläutert werden (mit $B_{x}, B_{y}$ und $B_{z}$ als Variablen für Buchstaben des lat. Alphabets):

(a) Transitivität: Für drei verschiedene Buchstaben des Alphabets $B_{x}, B_{y}, B_{z}$ gilt stets: Wenn $B_{x} B_{y}$ vorausgeht und wenn $B_{x} B_{z}$ vorausgeht, dann geht auch $B_{x} B_{z}$ voraus.

Beispiel: Wenn $\mathrm{a} b$ vorausgeht und wenn $\mathrm{b} \mathrm{z}$ vorausgeht, dann geht auch a $z$ voraus. Dem entspricht: Wenn 1 kleiner ist als 2 und wenn 2 kleiner ist als 26, dann ist auch 1 kleiner als 26 .

(b) Asymmetrie: Für zwei verschiedene Buchstaben des Alphabets $\mathrm{B}_{\mathrm{x}}, \mathrm{B}_{\mathrm{y}}$ gilt stets: Wenn $\mathrm{B}_{\mathrm{x}} \mathrm{B}_{\mathrm{y}}$ vorausgeht, dann geht $\mathrm{B}_{\mathrm{y}}$ nicht $\mathrm{B}_{\mathrm{x}}$ voraus.

Beispiel: Wenn $b$ d vorausgeht, dann geht $d$ nicht $b$ voraus. Dem entspricht: Wenn 2 kleiner ist als 4 , dann ist 4 nicht kleiner als 2.

(c) Konnexität: Für die zwei geordneten Paare von 2 Buchstaben des Alphabets $B_{x}, B_{y}$ gilt stets: Entweder ist $\left\langle B_{x}, B_{y}\right\rangle$ ein Element der alphabetischen Relation oder $\left\langle B_{y}, B_{x}\right\rangle$. Oder anders ausgedrückt: Zwei beliebige Buchstaben $B_{x}, B_{y}$ des Alphabets sind hinsichtlich der alphabetischen Relation vergleichbar.

Beispiel: Nimmt man die Buchstaben a und q und bildet die beiden möglichen Paare $\langle a, q\rangle$ und $\langle q, a\rangle$, dann ist nur $\langle a, q\rangle$ ein Element der Relation, da gilt: a geht $\mathrm{q}$ voraus. Dem entspricht: Nimmt man die geordneten Paare $\langle 1,16\rangle$ und $\langle 16,1\rangle$, dann ist nur das erstgenannte Paar ein Element der Kleiner-als-Relation.

Es ist klar, dass die soeben grob erläuterte Isomorphie eines Ausschnitts der Zahlenreihe mit dem lat. Alphabet die Grundlage dafür ist, dass man auf der Basis des Alphabets lineare Ordnungen herstellen kann. Das bedeutet aber nicht, dass Personen, wenn sie eine Menge von sprachlichen Ausdrücken alphabetisch sortieren, bewusst auf diese Isomorphie zurückgreifen. Günther (1996: 1568f) schreibt über das alphabetische Sortieren: 
Die Technik alphabetischen Sortierens und des Auffindens von Information in alphabetisch sortierten Listen ist den meisten literaten Menschen so vertraut, daß es gar nicht so einfach ist, sich klarzumachen, was dieser Technik und Tätigkeit zugrundeliegt; wissenschaftliche Literatur ist dazu kaum $\mathrm{zu}$ finden. Worin besteht eine vollständige alphabetische Anordnung, was macht man beim vollständigen, absoluten oder mechanischen alphabetischen sortieren (Wiegand 1989)? Die Elemente einer Liste, z.B. die Lemmata eines Wörterbuchs, werden beim absoluten Alphabetisieren analysiert als den jeweiligen Buchstabenketten zugeordnete Zahlenwerte. Dabei wird jeder Buchstabenposition des Wortes diejenige Ziffer zugeordnet, die der Stelle des Buchstabens in der Alphabetreihe entspricht.

Zwar ist es richtig, dass die Grundlagen des Alphabetisierens mathematisch expliziert werden können. Beim normalen alphabetischen Sortieren und beim externen Zugreifen auf alphabetische äußere Zugriffsstrukturen, bei dem ja das alphabetische Sortieren nachvollzogen wird, also insgesamt in allen praktischen Handlungszusammenhängen, in denen alphabetisiert wird, spielen die möglichen Zuordnungen von Buchstaben zu Zahlenwerten überhaupt keine Rolle. Günther verwechselt die extrasituative bewusstseinsfremde Perspektive eines Mathematikers bzw. eines Wissenschaftlers mit der intrasituativen bewusstseinseigenen Perspektive derjenigen, die alphabetisch sortieren und alphabetfundiert extern zugreifen möchten. Vielmehr gilt: In beiden Handlungssituationen greifen die Agenten lediglich in intuitiver Weise auf die erläuterten Eigenschaften der Ordnungsstruktur zurück. Entsprechend lässt sich das Alphabetisieren am einfachen Beispiel wie folgt erläutern: Die drei Wortformen Mona, Monist und Monster seien alphabetisch zu sortieren. Dann werden diese drei Wortformen Buchstabenposition für Buchstabenposition verglichen: Das Ergebnis ist: Die drei Wortformen sind in den ersten drei Buchstabenpositionen gleich (Mon). Also bestimmen die Buchstaben in der vierten Buchstabenposition ihre alphabetische Reihenfolge, und zwar gilt u.a.: Im Alphabet geht a i voraus; also kommt Mona vor Monist. Weiterhin gilt: Im Alphabet geht i s voraus; entsprechend kommt Monist vor Monster, so dass die lineare Ordnungsstruktur, die als Ergebnis des alphabetischen Sortierens zustande kommt, folgende Form aufweist: Mona < Monist < Monster (hier mit " $<$ " für geht voraus). Beim alphabetischen Sortieren ebenso wie beim alphabetfundierten externen Zugreifen auf alphabetische Zugriffsstrukturen benötigt man also keine Zahlen, sondern die Kenntnis des Alphabets und damit die intuitive Kenntnis der Ordnungsaxiome, die für das Alphabet als lineare Ordnungsstruktur gelten, sowie eine gewisse Übung, so dass man die allgemeine Wörterbuchbenutzungspraxis (sensu Wiegand 1998: 370) beherrscht.

Wir kennen jetzt diejenigen ordnungstheoretischen Eigenschaften eines Alphabets, deren Kenntnis die Voraussetzung dafür ist, dass man genau versteht, was die zentralen Eigenschaften einer alphabetischen äußeren Zugriffsstruktur in ordnungstheoretischer Hinsicht sind. Für eine alphabetische äußere $\mathrm{Zu}-$ griffsstruktur gelten die gleichen Ordnungsaxiome wie für das Alphabet, rela- 
tiv zu dem sie erzeugt wurde. Entsprechend ist eine alphabetische äußere Zugriffsstruktur eine Menge, sie heiße $\mathrm{M}_{\mathrm{z}}$, von sprachlichen äußeren Zugriffstextelementen, auf der eine zweistellige alphabetische (a) Ordnungsrelation $\mathrm{R}_{\mathrm{a}} \subseteq \mathrm{M}_{\mathrm{z}}$ $\mathrm{x}_{\mathrm{z}}$ definiert ist, die transitiv, asymmetrisch und konnex ist. Eine alphabetische äußere Zugriffsstruktur ist mithin eine lineare Ordnungsstruktur. Dadurch ist es möglich, dass ein Benutzer, der die nötigen Kenntnisse hat, auf jedes sprachliche äußere Zugriffstextelement direkt extern zugreifen kann. Direkt extern auf eine alphabetische äußere Zugriffsstruktur zugreifen zu können, heißt dann - informell und vereinfacht ausgedrückt - anhand der Buchstabenfolge eines sprachlichen Ausdrucks, die Leitelement heißt (vgl. Abb. 3), z.B. anhand von |Mona |, auf der Basis der Kenntnis einer wörterbuchexternen linearen Ordnungsstruktur, nämlich der Kenntnis des Alphabets, ein alphabetisch eingeordnetes äußeres Zugriffstextelement, z.B. das Lemma Mona, in einer wörterbuchinternen linearen Ordnungsstruktur, welche die gleichen Ordnungsaxiome erfüllt wie das Alphabet, Ausdrücke gezielt suchen und finden zu können bzw. sicher entscheiden zu können, dass ein äußeres Zugriffstextelement, das die gleiche Buchstabenfolge aufweist wie das Leitelement, kein Element der sprachlichen äußeren Zugriffsstruktur ist und daher nicht gefunden werden kann, weil eine Lemmalücke eines bestimmten Typs vorliegt.

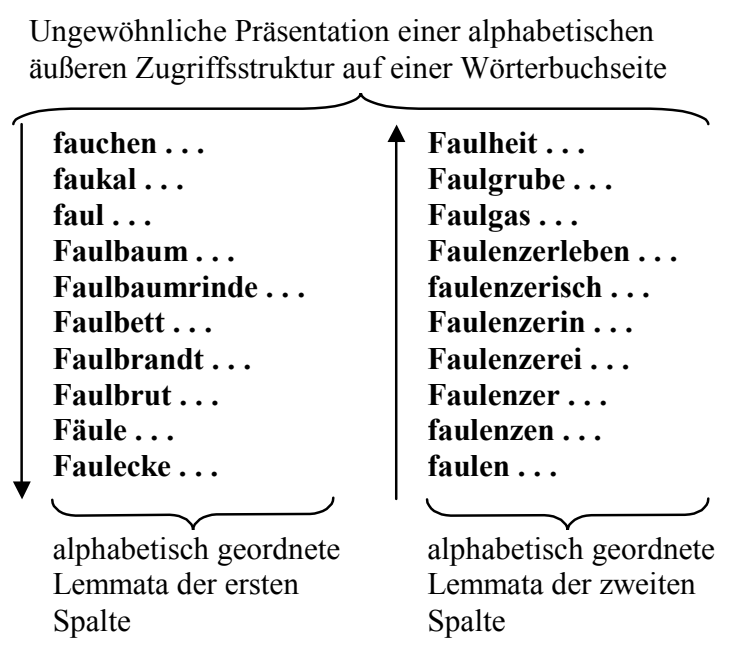

Abb. 2: Veranschaulichung zu einer ungewöhnlichen Präsentation einer alphabetischen äußeren Zugriffsstruktur im zweidimensionalen Druckraum

Bisher haben wir uns mit dem Ordnungsaspekt des Alphabets und der alphabetischen äußeren Zugriffsstrukturen befasst. Zugriffsstrukturen jeder Art müssen jedoch im zweidimensionalen Printraum auch präsentiert werden. Diese Präsentation richtet sich in der Regel nach Konventionen des jeweiligen Schriftsystems (vgl. zum Folgenden Wiegand 1989: 381). Für das Deutsche 
heißt dies, dass für ein normales Schriftstück oder Druckwerk gilt: Es wird auf (oder: in) Zeilen von links nach rechts geschrieben und auch so gelesen. Werden mehr als eine Zeile benötigt, dann wird nacheinander auf jeder Zeile von links nach rechts weiter geschrieben, und auf diese Weise wird dann auch von oben nach unten geschrieben. Auf der nächsten Seite geht es dann genau so weiter. Die Präsentation von alphabetischen Zugriffsstrukturen richtet sich nach diesen Konventionen. Das erkennt man sozusagen "auf einen Blick“, wenn man die Abb. 2 genauer betrachtet. In dieser ist in der ersten Spalte diese Konvention eingehalten. Die alphabetisch sortierte Lemmareihe läuft von oben nach unten. In der zweiten Spalte läuft sie dagegen von unten nach oben. Niemand wird behaupten wollen, die Lemmata seien deswegen insgesamt nicht alphabetisch sortiert, und selbstverständlich liegt auch eine alphabetische äußere $\mathrm{Zu}$ griffsstruktur vor, diese ist lediglich im zweidimensionalen Printraum auf eine Weise präsentiert, die nach dem deutschen Schriftsystem nicht vorgesehen ist.

Das Beispiel in Abb. 2 lehrt: (1) Die Ordnungseigenschaften einer alphabetischen äußeren Zugriffsstruktur sind von ihrer zweidimensionalen Präsentation unabhängig. (2) Da es im Rahmen der Schriftsystemkonventionen eine Reihe von Möglichkeiten gibt, die äußeren Zugriffstextelemente zu präsentieren, lassen sich alphabetische äußere Zugriffsstrukturen auch nach der Art ihrer Präsentation und damit nach den Lageeigenschaften der äußeren Zugriffstextelemente im zweidimensionalen Druckraum unterscheiden. Dies kann hier nur an einem Beispiel anhand der Abb. 3 erläutert werden.

In der Abb. 3 wird u.a. veranschaulicht, dass auf der (nur ausschnittsweise dargestellten) Menge aller äußeren Zugriffstextelemente, die im vorliegenden Fall Lemmata sind, nicht nur eine zweistellige Ordnungsrelation vom Typ der alphabetischen Ordnungsrelation definiert ist (auf deren Elemente der Relationsterm $x$ geht $y$ alphabetisch voraus zutrifft), sondern auch eine zweistellige texttopologische (oder: textarchitektonische) Ordnungsrelation, nämlich eine vom Typ der oberhalb-Relation (auf deren Elemente der Relationsterm $x$ ist (senkrecht) oberhalb von y zutrifft). Auch Relationen dieses Typs sind totale Ordnungsrelationen, die transitiv, asymmetrisch und konnex sind. Es gilt jetzt am Beispiel zweier Lemmata aus Abb. 3 erläutert - nicht nur „Mona geht alphabetisch Monist voraus", sondern auch "Mona ist senkrecht oberhalb (kurz: oberhalb) von Monist". Ist $\mathrm{M}_{\mathrm{L}}$ die Menge aller Lemmata (L) eines Wörterbuchs, dann gilt: Für alle Paare, die mit Lemmata $\mathrm{L}_{\mathrm{x}}, \mathrm{L}_{\mathrm{y}}$ aus $\mathrm{M}_{\mathrm{L}}$ gebildet werden können, entweder: Auf das Paar $\left\langle\mathrm{L}_{\mathrm{x}}, \mathrm{L}_{\mathrm{y}}\right\rangle$ trifft sowohl der Relationsterm $L_{x}$ geht alphabetisch $L_{y}$ voraus $\mathrm{zu}$, als auch der Relationsterm $L_{x}$ ist oberhalb von $L_{y}$; oder es gilt: Auf das Paar $\left\langle\mathrm{L}_{\mathrm{y}}, \mathrm{L}_{\mathrm{x}}\right\rangle$ trifft sowohl der Relationsterm $L_{y}$ geht alphabetisch $L_{x}$ voraus $\mathrm{zu}$, als auch der Relationsterm $L_{y}$ ist oberhalb von $L_{x}$. Oder anders ausgedrückt: Zwei beliebige Lemmata $L_{x}, L_{y}$ der Menge $M_{L}$ sind immer sowohl hinsichtlich der alphabetischen Relation als auch hinsichtlich der oberhalb-Relation vergleichbar. Nehmen wir als Beispiel: $\mathrm{L}_{\mathrm{x}}=$ Mona und $\mathrm{L}_{\mathrm{y}}=$ Monist, dann ist offenbar das geordnete Paar 〈Mona, Monist〉 und nicht das geordnete Paar 〈Monist, Mona〉 ein Element beider zweistelligen Ordnungsrelationen. 


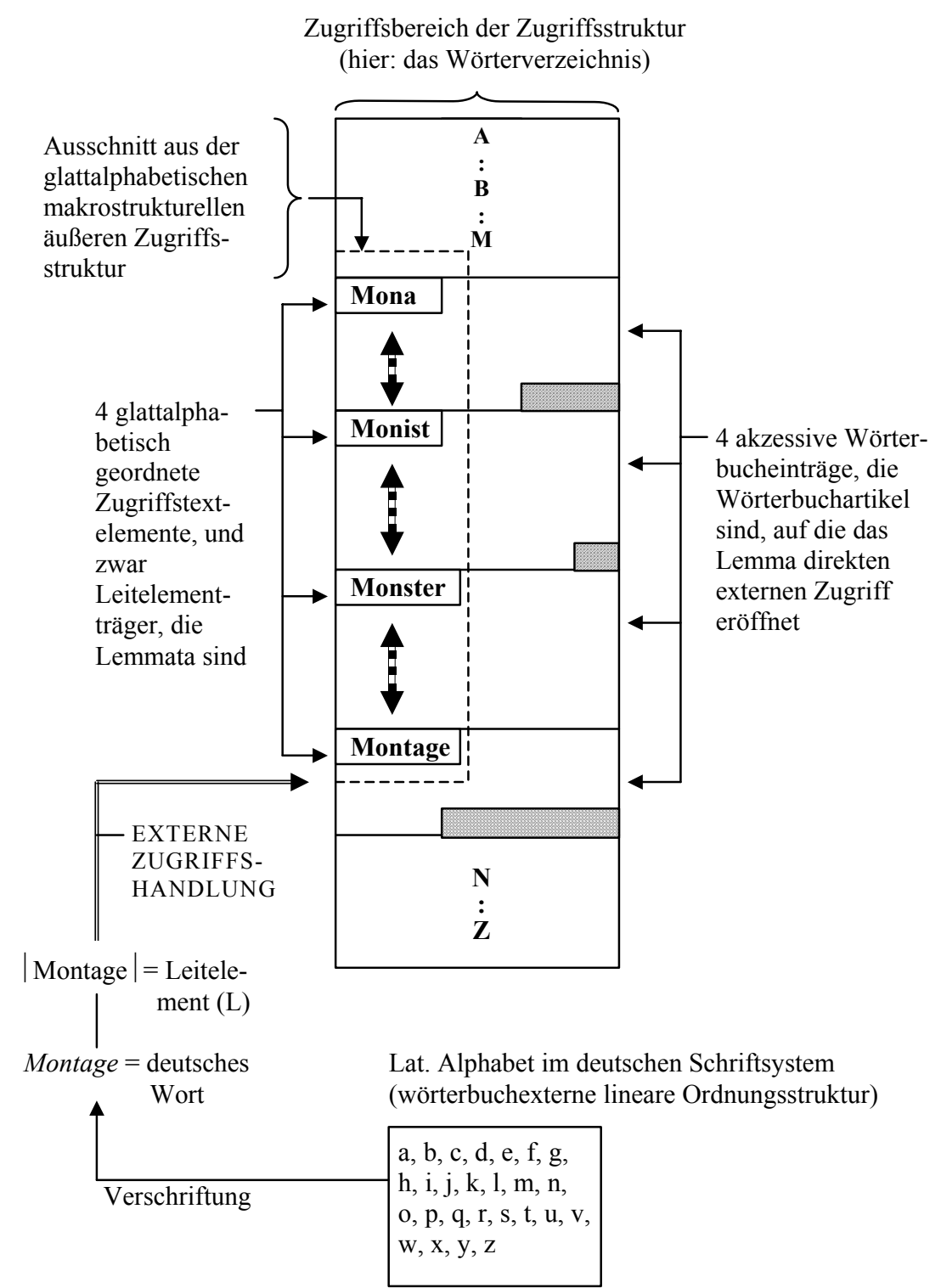

Abb. 3: Veranschaulichung zur externen Datenakzessivität, die mittels einer glattalphabetischen makrostrukturellen äußeren Zugriffsstruktur hergestellt ist. Der Doppelpfeil bedeutet soviel wie $x$ ist oberhalb von $y$, mit " $\mathrm{x}^{\prime \prime}$ und "y" als Variablen für äußere Zugriffstextelemente

Wir können nun also feststellen: Ist auf der Menge aller Lemmata eines Wörterbuchs sowohl eine zweistellige Ordnungsrelation vom Typ der alphabetischen 
als auch eine vom Typ der oberhalb-Relation definiert, dann liegt eine glattalphabetische makrostrukturelle äußere Zugriffsstruktur vor.

Ist auf der Menge aller Lemmata keine oberhalb-Relation definiert, dann liegt eine nicht glattalphabetische makrostrukturelle äußere Zugriffsstruktur vor. Zugriffsstrukturen dieses Typs, die nicht zu einem seiner speziellen Untertypen gehören - wie z.B. die nischenalphabetischen makrostrukturellen Zugriffsstrukturen - kommen kaum vor, weil sie extrem benutzerunfreundlich sind. Bei den alphabetischen makrostrukturellen äußeren Zugriffsstrukturen lässt sich die für die Typen von Makrostrukturen entwickelte Terminologie (vgl. Wiegand 1989; 1998a; 2002; Bergenholtz, Tarp und Wiegand 1999: 1811ff) weitgehend übernehmen. Dies sei wenigstens an einem Beispiel demonstriert. In Abb. 4 findet sich ein Ausschnitt aus dem Wörterverzeichnis des Duden-2 $\mathrm{GW}$.

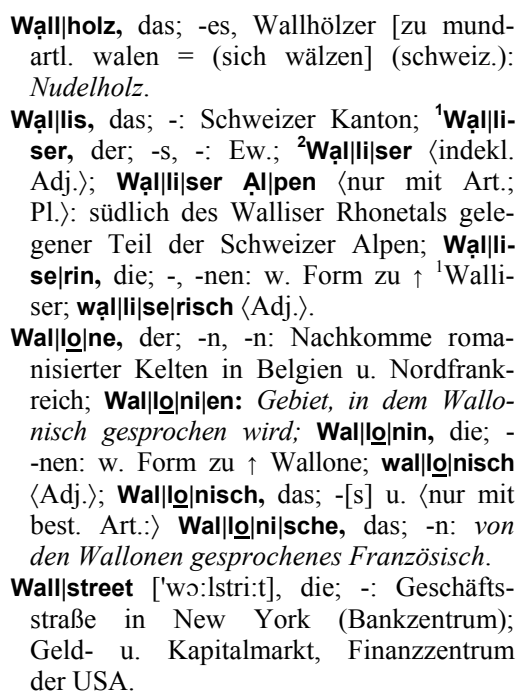

\section{Abb. 4: Ausschnitt aus dem Wörterverzeichnis des Duden-2W}

Der Ausschnitt in Abb. 4 beginnt mit einem Einzelartikel zum Lemmazeichen Wallholz. Es folgen zwei gruppierte Artikelnischen. Die erste Nische beginnt mit dem Nischeneingangslemma Wal lis; die zugehörige unten und binnenerweiterte Lemmazeichengestaltangabe ist $\mathbf{W}$ ạl|lis. Die zweite Nische beginnt mit dem Nischeneingangslemma Wal lo ne; die zugehörige unten und binnenerweiterte Lemmazeichengestaltangabe ist $\mathbf{W a l}|\mathbf{l} \underline{\mathbf{o}}|$ ne. In der ersten gruppierten Artikelnische stehen fünf, in der zweiten stehen ebenfalls fünf gruppierte Nischenlemmata. Auf einer Teilmenge der Menge aller Lemmata des Duden${ }^{2} \mathrm{GW}$, nämlich der Menge derjenigen Lemmata, die keine gruppierten Nischenlemmata sind, ist eine oberhalb-Relation definiert; entsprechend liegt eine komprimiert-nischenalphabetische makrostrukturelle Zugriffsstruktur vor. Eine Veranschaulichung zu diesem Zugriffsstrukturtyp findet sich in der Abb. 5. 


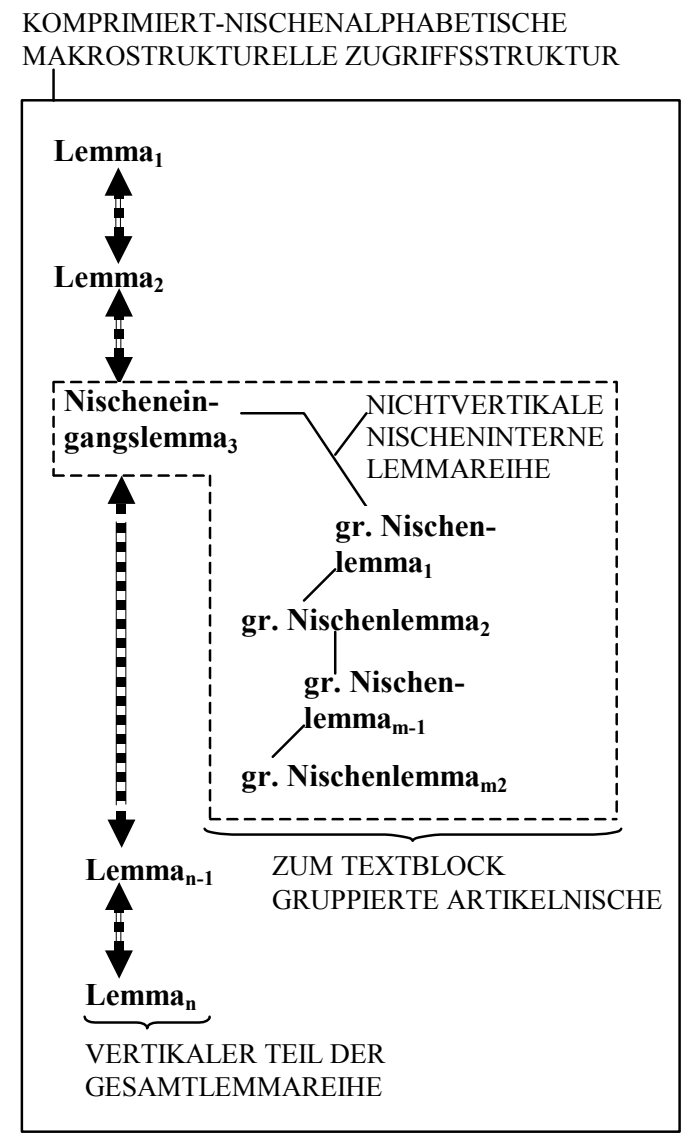

Abb. 5: Veranschaulichung zur komprimiert-nischenalphabetischen makrostrukturellen Zugriffsstruktur

Es ist oben schon darauf hingewiesen worden, dass das lat. Alphabet nicht ausreicht, um alle in deutschen Texten vorkommende Wörter so alphabetisch zu sortieren, dass eine lineare Ordnungsstruktur gegeben ist. Denn in den Leitelementen, die zu deutschen Wörtern gehören, können neben den 26 alphabetinternen Buchstaben weitere alphabetexterne, aber schriftsysteminterne Buchstaben auftreten, nämlich die deutschen Zusatzbuchstaben ä, ö, ü und $B$ sowie darüberhinaus schriftsystemexterne Buchstaben. Weiterhin können numerische Elemente auftreten, das Leerzeichen muss - je nach gewählter Alphabetisierungsmethode - in verschiedener Weise berücksichtigt werden u.v.a.m. Das lat. Alphabet zusammen mit der Menge aller Zusatzregelungen ergibt dann das je wörterbuchspezifische Zugriffsalphabet, das in einem der Metatexte erklärt werden muss (vgl. Wiegand 1989: 376ff). 
Entsprechend lassen sich dann der uneingeschränkte Zugriff von dem eingeschränkten Zugriff auf alphabetische Zugriffsstrukturen unterscheiden. Ein uneingeschränkter Zugriff im Zuge der Ausführung einer externen Zugriffshandlung (sensu Wiegand 1998: 393ff) ist möglich, wenn das Leitelement nur aus alphabetinternen Buchstaben besteht. Besteht dagegen das Leitelement nicht nur aus alphabetinternen Buchstaben, wie dies z.B. bei folgenden Leitelementen der Fall ist: |öde |, |küssen |, |Bär|, |weiß| oder soll auf Bezeichnungen, wie z.B. $\alpha$-Strahlung oder 8-Azaguanin zugegriffen werden, bei denen nicht ohne weiteres klar ist, was zum Leitelement gehört, dann ist nur ein eingeschränkter Zugriff möglich. Die Einschränkung besteht darin, dass die Kenntnis des lat. Alphabets nicht ausreicht, so dass der Benutzer nicht extern zugreifen kann, ohne über ein Zusatzwissen zum wörterbuchspezifischen Zugriffsalphabet zu verfügen, das anhand der Metatexte des Wörterbuchs erworben wurde.

Damit sei der erste Einblick, der uns zu dem im Schriftkulturraum der Alphabetschriften bekanntesten Typ der äußeren Zugriffsstrukturen führte, nämlich zum Typ der alphabetischen Zugriffsstrukturen, abgebrochen.

\subsection{Zweiter Einblick: Weitere Typen von äußeren Zugriffsstrukturen}

Im Folgenden betrachten wir weitere Typen von äußeren Zugriffsstrukturen, und zwar solche, die nicht - wie die glattalphabetischen makrostrukturellen und die komprimiert-nischenalphabetischen makrostrukturellen Zugriffsstrukturen - zu einem Untertyp des Typs der alphabetischen makrostrukturellen äußeren Zugriffsstrukturen gehören. Dazu gehen wir von dem einfachsten Fall, nämlich den monoakzessiven Einbändern ohne Verweisungen, zu einem komplexeren Fall über: Wir berücksichtigen jetzt Wörterbücher, die integrierte Umtexte aufweisen und in denen Verweisungen anzutreffen sind.

Um zu verstehen, wie Zugriffsstrukturen funktionieren, die nicht zu den alphabetischen makrostrukturellen äußeren Zugriffsstrukturen gehören und weiterhin für das Verständnis der Bifunktionalität von alphabetischen makrostrukturellen äußeren Zugriffsstrukturen in solchen Wörterbüchern, in denen lemmatisch orientierte Verweisungen vorgenommen wurden, muss zunächst in vereinfachter Form erläutert werden, was unter einer externen Zugriffshandlung und unter einem externen Zugriff zu verstehen ist. Selbst wenn eine solche Erläuterung hier nur sehr grob ausfallen kann, lässt sie sich nicht ohne einen Rückgriff auf handlungstheoretische Grundeinsichten geben (vgl. Wiegand 1998: 270-292). Zunächst sollte das Folgende klar sein: Eine Handlung $h_{1}$, die zu einem bestimmten Handlungstyp $\mathrm{HT}_{1}$ gehört, wird stets dadurch ausgeführt, dass mindestens eine Handlung $\mathrm{h}_{2}$ ausgeführt wird, die zu einem bestimmten anderen Handlungstyp $\mathrm{HT}_{2}$ gehört. Hierzu ein Beispiel aus der Grundschule: Eine Handlung $\mathrm{h}_{3}$ vom Typ EINEN BRUCH DURCH EINEN ANDEREN BRUCH DIVIDIEREN $\left(=\mathrm{HT}_{3}\right)$ wird dadurch ausgeführt, dass eine Handlung $\mathrm{h}_{4}$ vom Typ EINEN BRUCH MIT DEM KEHRWERT DES ANDE- 
REN BRUCHES MULTIPLIZIEREN $\left(=\mathrm{HT}_{4}\right)$ ausgeführt wird. Man schreibt hierfür auf der generischen Ebene: $\left\{\mathrm{HT}_{3}\right\} \rightarrow\left\{\mathrm{HT}_{4}\right\}$; hier repräsentiert der Rechtspfeil " $\rightarrow$ " die dadurch-dass-Relation. Handlungstypen (und damit die zugehörigen individuellen Handlungen $h_{i}$ ), die unmittelbar rechts vom Rechtspfeil genannt werden (im Beispiel also $\mathrm{HT}_{4}$ ) liegen im Ausführungsbereich des Handlungstyps, der unmittelbar vor dem Rechtspfeil genannt wird (im Beispiel $\mathrm{HT}_{3}$ ). Die dadurch-dass-Relation ist transitiv; entsprechend können mehrere Rechtspfeile hintereinander auftreten, so dass ein unmittelbarer und mindestens ein mittelbarer Ausführungsbereich gegeben sein können. Für den vorliegenden Zusammenhang ist es nun besonders wichtig zu berücksichtigen, dass externe Zugriffshandlungen im unmittelbaren Ausführungsbereich von usuellen Benutzungshandlungen liegen können, die zu zwei unterschiedlichen Typen von Benutzungshandlungen gehören. Dagegen ist hier nicht wichtig, wie externe Zugriffshandlungen genau ausgeführt werden; d.h.: Die Handlungstypen (und die zugehörigen Handlungen), die im Ausführungsbereich von externen Zugriffshandlungen liegen, sind hier nicht von Interesse. Im Folgenden nehmen wir als Beispielwörterbuch Wahrig (1989). Dessen vollständig kondensierte Wörterbuchartikel (sensu Wiegand 2003: 207f) weisen innere Zugriffsstrukturen auf (zu diesen vgl. 4). Eine Nachschlagehandlung anhand von Wörterbüchern, die innere Zugriffsstrukturen aufweisen, kann dann vereinfacht wie folgt charakterisiert werden: Eine Nachschlagehandlung, die zum Handlungstyp IN EINEM WÖRTERBUCH ETWAS NACHSCHLAGEN (= HT6) gehört, ist eine Benutzungshandlung, die dadurch ausgeführt wird, dass nach der erfolgreichen Ausführung einer Handlung vom Typ AUF EIN WÖRTERBUCH EXTERN ZUGREIFEN (= HT-10) eine Handlung vom Typ AUF DIE DATEN EINES AKZESSIVEN WÖRTERBUCHEINTRAGES INTERN ZUGREIFEN (= HT-7) ausgeführt wird ( $\{$ HT-6 $\} \rightarrow\{$ erst: HT-10, dann: HT-7\}).

Hier steht also der Typ der externen Zugriffshandlung (HT-10) im unmittelbaren Ausführungsbereich des Typs der Nachschlagehandlung (HT-6). Wer also z.B. (vgl. Abb. 3) nicht weiß, was Montage bedeutet und deswegen im Wörterbuch nachschlagen möchte, der muss dies dadurch tun, dass er - ausgestattet mit dem Leitelement |Montage | - zuerst eine externe Zugriffshandlung erfolgreich ausführt, so dass er das Lemma Montage gefunden hat. Als externer Zugriff gilt der erfolgreiche Abschluss der Ausführung einer externen Zugriffshandlung, der gerade darin besteht, dass ein Benutzer das äußere Zugriffstextelement gefunden hat. Erst danach kann er eine interne Zugriffshandlung ausführen, die ihm zur Bedeutungsparaphrasenangabe im Wörterbuchartikel zu Montage führt.

Anders verhält es sich dagegen, wenn ein Benutzer im gleichen Wörterbuch im Wörterbuchartikel zu Zaun ein verweisvermittelndes Textsegment findet, z.B. die lemmatisch orientierte vollständige Verweisangabe " $\rightarrow$ Montage", die aus der Verweisbeziehungsangabe $" \rightarrow$ " und der Angabe der lemmatischen Verweisaußenadresse „Montage“ besteht (vgl. Abb. 6). 


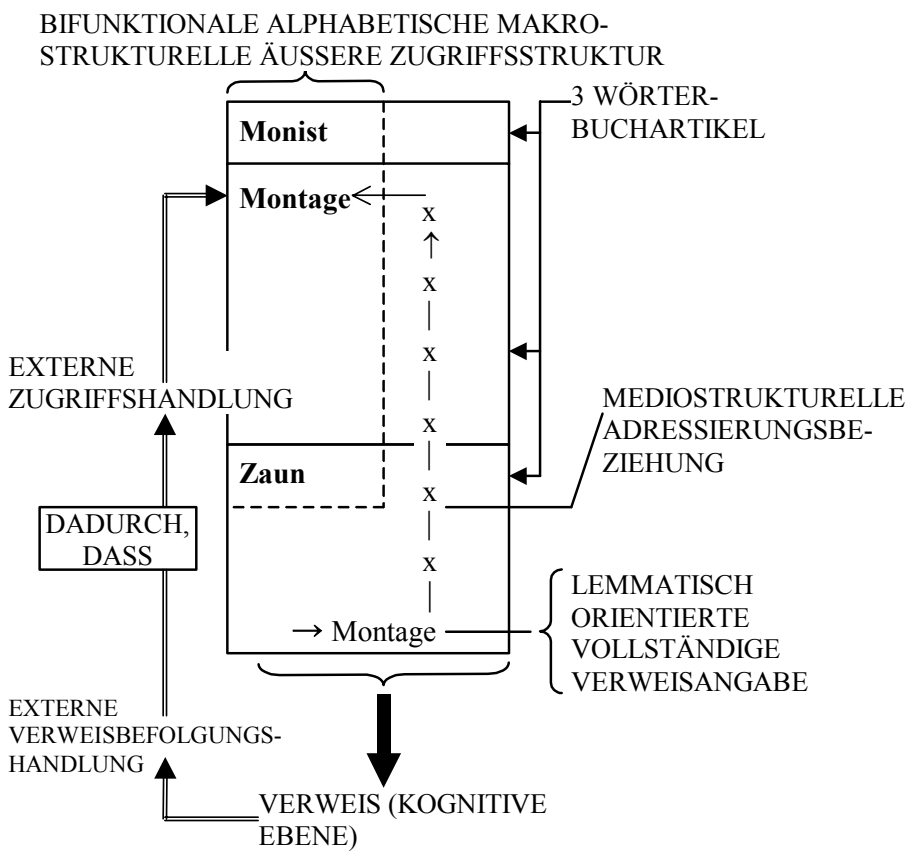

Abb. 6: Veranschaulichung zur Bifunktionalität alphabetischer makrostruktureller äußerer Zugriffsstrukturen; $\mathrm{A} \rightarrow \mathrm{B}$ bedeutet soviel wie anhand von $A$ wird $B$ erschlossen.

Dieser Benutzer kann dann anhand der Verweisangabe " $\rightarrow$ Montage" einen Verweis erschließen; dieser besteht in seinem Wissen, dass er, um das Verweisziel zu erreichen, eine externe Verweisbefolgungshandlung ausführen muss, und zwar mit Hilfe der im Artikel zu Zaun genannten Verweisadresse | Montage $\mid$, die damit als mediostrukturelles Leitelement fungiert. Die Ausführung der externen Verweisbefolgungshandlung kann nur dadurch geschehen, dass der Benutzer eine externe Zugriffshandlung ausführt, die ihn zum Lemma Montage führt. Das Lemma ist damit nicht nur ein Leitelementträger, sondern auch ein Verweisadressenträger. Damit sollte deutlich sein, dass es auch externe Zugriffshandlungen gibt, die im unmittelbaren Ausführungsbereich von Verweisbefolgungshandlungen liegen. Während bei externen Zugriffshandlungen, die im Ausführungsbereich von Nachschlagehandlungen liegen, das zur Handlungsausführung benötigte Leitelement wörterbuchextern erschlossen wird, wird bei externen Zugriffshandlungen, die im Ausführungsbereich von Verweisbefolgungshandlungen liegen, das zur Handlungsausführung benötigte Leitelement, nämlich die jeweilige Verweisadresse, anhand von verweisvermittelnden Textsegmenten und damit wörterbuchintern erschlossen. Aus diesem Grunde heißen solche Leitelemente mediostrukturelle Leitelemente. In einem Wörterbuch, 
in dem lemmatische Verweisaußenadressen, wie z.B. |Montage $\mid$ mit verweisvermittelnden Textsegmenten, wie z.B. der vollständigen Verweisangabe " $\rightarrow$ Montage", genannt werden, fungiert die alphabetische makrostrukturelle äußere Zugriffsstruktur immer auch als mediostrukturelle äußere Zugriffsstruktur, also als Fundortdomäne, in der die Verweisaußenadressen zu finden sind. Sie ist damit bifunktional. Dies ist in modernen Printwörterbüchern der Normalfall, wurde aber bisher in der Wörterbuchforschung kaum explizit dargestellt.

Im Folgenden soll erläutert werden, was eine mediostrukturelle äußere Zugriffsstruktur ist. Dies geschieht anhand von Abb. 7.

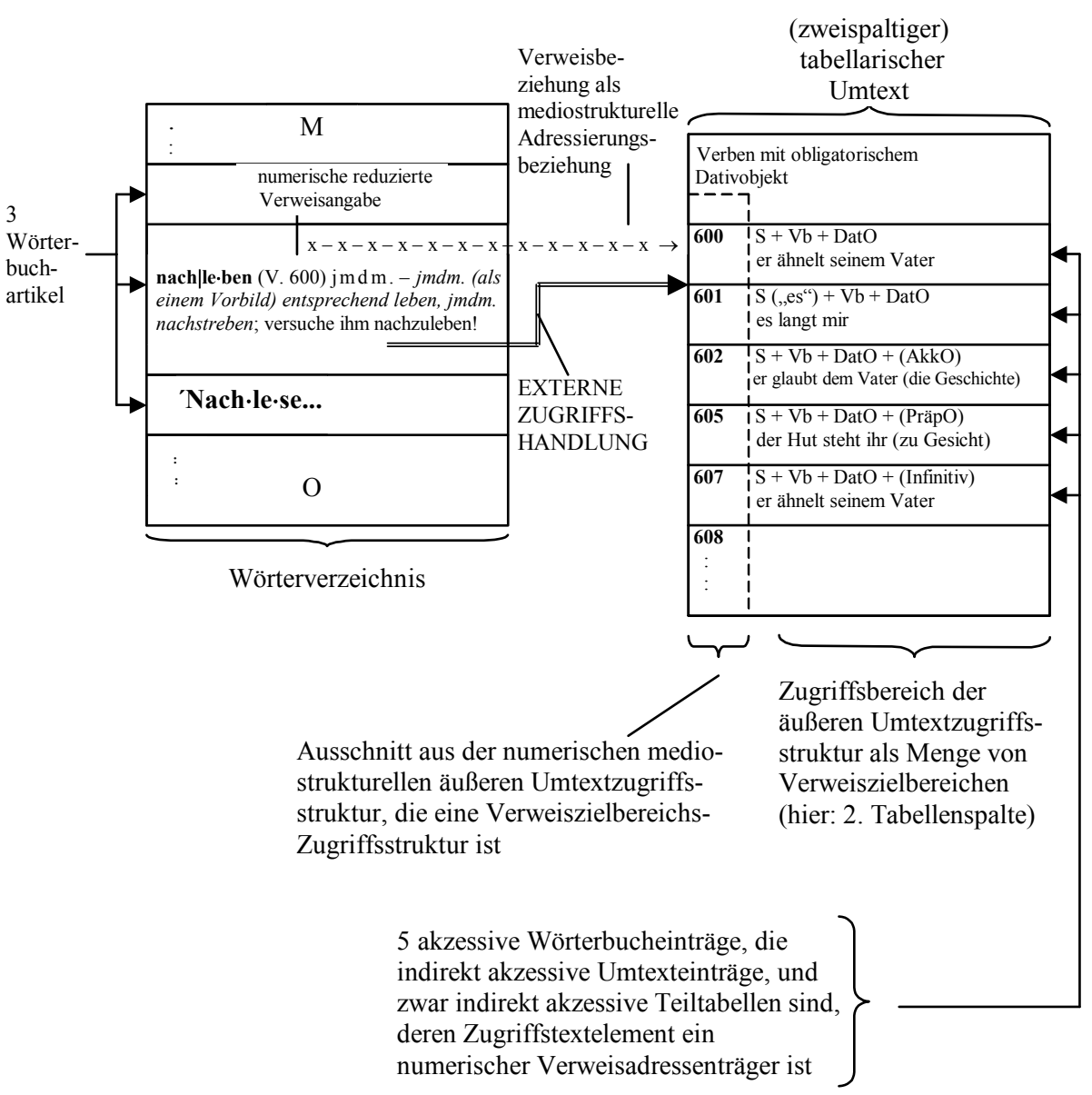

Abb. 7: Veranschaulichung zu mediostrukturellen äußeren Zugriffsstrukturen 
Die Abb. 7 zeigt ein Beispiel aus dem Wahrig (1989). Links ist ein Ausschnitt aus dem Wörterverzeichnis zu sehen, rechts ein Ausschnitt aus einem zweispaltigen tabellarischen Umtext, der sich im Vorspann des Wörterbuchs findet. Im Artikel zum Lemmazeichen nachleben findet sich nach der verdichteten Wortartangabe „V.“, der Abkürzung für Verb, eine numerische reduzierte Verweisangabe, nämlich "600"; reduziert ist sie deswegen, weil sie keine Verweisbeziehungsangabe als Teilangabe aufweist. Die reduzierte Verweisangabe ist umtextorientiert: Der Adressenfundort der genannten Verweisadresse |600| ist die numerische äußere Umtextzugriffsstruktur, von der der Ausschnitt $<600$, 601, 602, 605, 607, 608> in Abb. 7 zu sehen ist; sie bildet die erste Spalte des tabellarischen Umtextes, die damit eine Zugriffsspalte ist. Es besteht mithin eine mediostrukturelle Adressierungsbeziehung von der reduzierten Verweisangabe "600“ im Artikel zu nachleben zum Verweisadressenträger "600" in der numerischen äußeren Umtextzugriffsstruktur. Auf letztere kann nur im Zuge der Ausführung von externen Zugriffshandlungen zugegriffen werden, die im unmittelbaren Ausführungsbereich von externen Verweisbefolgungshandlungen liegen. Zwar kann man in den tabellarischen Umtexten des Wahrig (1989) "herumlesen" und sich z.B. darüber beim Lesen informieren, wieviele und welche Typen von Satzmusterangaben im Wahrig (1989) vorgesehen sind. Bei einer solchen Wörterbuchbenutzung werden aber keine externen Zugriffshandlungen anhand eines nichtmediostrukturellen Leitelements ausgeführt, das wörterbuchextern gegeben ist. Für die numerische äußere Umtextzugriffsstruktur, von der in Abb. 7 ein Ausschnitt wiedergegeben ist, gilt Folgendes: Weil auf sie ausschließlich mit Hilfe eines mediostrukturellen Leitelements, das wörterbuchintern gegeben ist, im Zuge der Ausführung einer externen Zugriffshandlung zugegriffen werden kann, die im Ausführungsbereich einer externen Verweisbefolgungshandlung liegt, gehört sie zu einem bestimmten Typ der mediostrukturellen äußeren Zugriffsstrukturen.

Unter dem Aspekt der externen Datenakzessivität muss der Zugriffsbereich der äußeren Umtextzugriffsstruktur (also die gesamte zweite Spalte des zweispaltigen tabellarischen Umtextes; vgl. Abb. 7) als eine Menge von Verweiszielbereichen betrachtet werden. Die einzelnen Umtexteinträge in Abb. 7, also z.B.

$600 \mathrm{~S}+\mathrm{Vb}+\mathrm{DatO}$

er ähnelt seinem Vater

fungieren nur als Verweiszielbereiche, d.h.: Auf sie kann - wie wir ja bereits gesehen haben - nur im Zuge der Ausführung von Verweisbefolgungshandlungen extern zugegriffen werden. Aus diesem Grund gehört die numerische mediostrukturelle äußere Umtextzugriffsstruktur zu dem Untertyp der mediostrukturellen Zugriffsstrukturen, die Verweiszielbereichs-Zugriffsstrukturen heißen. Der zweite wichtige Untertyp der mediostrukturellen Zugriffsstrukturen sind die adressenvermittelnden Zugriffsstrukturen; auf diese wird unter 3.3 eingegangen. 
Bereits unter 1. wurde darauf hingewiesen, dass nicht alle äußeren $\mathrm{Zu}$ griffsstrukturen solche sind, auf die man von außerhalb eines Wörterbuches kommend zugreifen kann. Wir haben jetzt die begrifflichen Mittel zur Verfügung, um dies genauer auszuführen. Anhand der Abb. 3 hatten wir gesehen, dass man auf eine alphabetische makrostrukturelle äußere Zugriffsstruktur „Von außerhalb des Wörterbuchs kommend" (also mit Hilfe eines nichtmediostrukturellen Leitelements) extern zugreifen kann. Anhand der Abb. 6 hatten wir gesehen, dass man auf eine alphabetische makrostrukturelle äußere $\mathrm{Zu}$ griffsstruktur auch ",von innerhalb des Wörterbuchs kommend" (also mit Hilfe eines mediostrukturellen Leitelements) extern zugreifen kann. Schließlich haben wir anhand der Abb. 7 gesehen, dass man auf eine numerische äußere Umtextzugriffsstruktur nur ",von innerhalb des Wörterbuchs kommend" (also nur mit Hilfe eines mediostrukturellen Leitelements) extern zugreifen kann. Damit ist zunächst deutlich, dass äußere Zugriffsstrukturen (von welchem Untertyp auch immer) nicht dadurch definiert werden können, dass man sagt, der externe Zugriff erfolge „,von außerhalb des Wörterbuchs". Vielmehr ist das entscheidende Definitionskriterium dafür, dass eine Zugriffsstruktur eine äußere Zugriffsstruktur ist, der Sachverhalt, dass auf eine äußere Zugriffsstruktur (beliebig oft) extern zugegriffen werden kann, und zwar sowohl im Zuge von Nachschlagehandlungen (,von außerhalb des Wörterbuchs kommend“) als auch im Zuge von Verweisbefolgungshandlungen (,,von innerhalb des Wörterbuchs kommend").

Verfügt man über den Begriff des äußeren (oder: externen) Zugriffspfads, lässt sich auch wie folgt argumentieren: Eine äußere Zugriffsstruktur ist eine Zugriffsstruktur, zu der von Benutzern beliebig viele äußere Zugriffspfade etabliert werden können. Ein äußerer Zugriffspfad ist der je individuell etablierte, durch die Reihe der äußeren Zugriffstextelemente (z.B. die Lemmareihe) führende Weg, den ein Benutzer-in-actu gegangen ist, wenn er eine externe $\mathrm{Zu}$ griffshandlung erfolgreich ausgeführt hat, so dass diese mit dem externen Zugriff auf ein äußeres Zugriffstextelement (als Element der äußeren Zugriffsstruktur) endet. Jeder einfache (i.S.v. nicht zusammengesetzte) äußere Zugriffspfad hat einen Pfadanfang und ein Pfadende. Als Pfadanfang gilt der Ort, an dem das Leitelement erfasst wird. Als Pfadende gilt das zugehörige äußere Zugriffstextelement. Alle Pfadenden sind damit wörterbuchintern. Der Pfadanfang kann dagegen wörterbuchextern oder wörterbuchintern situiert sein. Ersteres ist genau dann der Fall, wenn die pfadetablierende externe Zugriffshandlung im Ausführungsbereich einer Nachschlagehandlung liegt; letzteres ist dagegen immer dann der Fall, wenn die pfadetablierende externe Zugriffshandlung im Ausführungsbereich einer Verweisbefolgungshandlung liegt. Entsprechend lassen sich zwei Typen von einfachen äußeren Zugriffspfaden unterscheiden, und zwar Folgende:

(a) Der Typ des adkurrenten äußeren Zugriffspfades. Dies ist ein Zugriffspfadtyp, zu dem alle einfachen äußeren Zugriffspfade gehören, deren Pfadanfang wörterbuchextern und deren Pfadende wörterbuchintern situiert ist. 
(b) Der Typ des binnenkurrenten äußeren Zugriffspfades. Dies ist ein Zugriffspfadtyp, zu dem alle einfachen äußeren Zugriffspfade gehören, deren Pfadanfang und Pfadende wörterbuchintern situiert sind. Die zuletzt eingeführten Begrifflichkeiten sind in Abb. 8 in einen Zusammenhang gebracht.

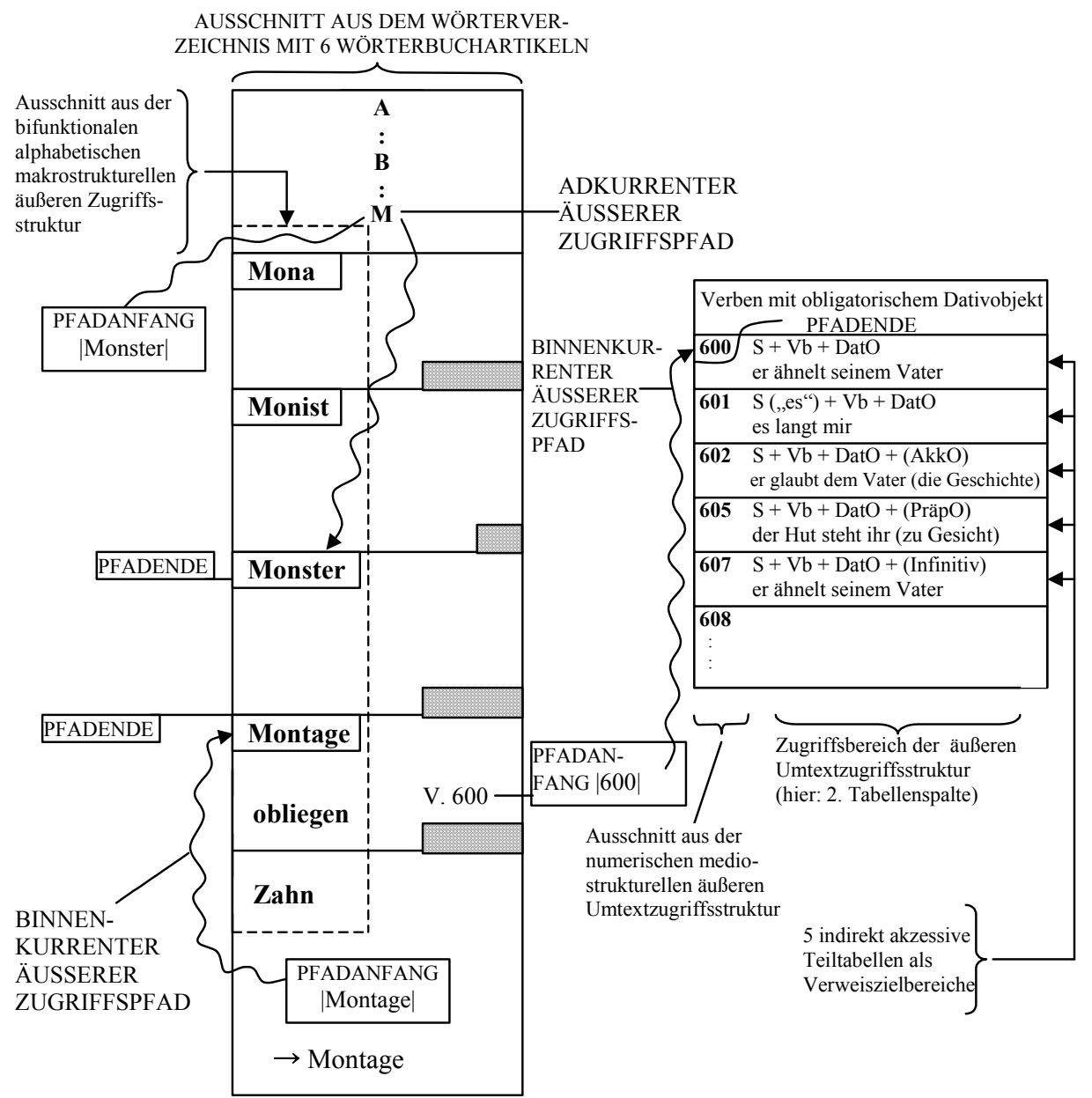

Abb. 8: Veranschaulichung zu äußeren Zugriffspfaden bei äußeren Zugriffsstrukturen

Hier sei noch ergänzt, dass Zugriffspfade auch Suchpfade genannt werden (vgl. Hausmann und Wiegand 1989: 337f). ${ }^{6}$ Unabhängig davon, ob von Zugriffspfaden oder von Suchpfaden gesprochen wird, gilt: Sie dürfen nicht mit Zugriffsstrukturen verwechselt werden. Leider ist dies in der Literatur nach Hausmann und Wiegand (1989) und Wiegand (1989), wo zum ersten Mal Zugriffsstruk- 
turen erörtert und von Suchpfaden deutlich unterschieden wurden, öfters der Fall. So beispielsweise in Bergenholtz und Tarp (1995: 16 u. 219ff) oder im Anschluss an diese bei Steyn (2004: 276), wo es heißt: "The access structure can be regarded as the search route the dictionary user follows during a dictionary consultation procedure."

Diese Feststellung ist nicht vereinbar mit der Folgenden: „In most general monolingual dictionaries, the central list is the only outer access structure; therefore the macrostructure of these dictionaries coincides with the outer access structure" (Steyn 2004: 280). Hier nun ist die äußere Zugriffsstruktur nicht mehr der Suchpfad (search route) sondern eine textuelle Struktur, die mit der Makrostruktur zusammenfällt, wobei noch die zusätzliche Verwechslung auftritt, dass das Wörterverzeichnis (central list) eine Zugriffsstruktur sein soll! Es sind mithin folgende Klarstellungen dringend erforderlich:

(a) Such- oder Zugriffspfade werden durch die Ausführung von Such- und Zugriffshandlungen etabliert und sind damit einmalige konkrete Phänomene. Äußere Zugriffspfade, die beim gleichen äußeren Zugriffstextelement enden, sind Elemente der gleichen Klasse von äußeren Zugriffspfaden.

(b) Äußere Zugriffsstrukturen sind dagegen nicht durch Handlungen konstituierte Phänomene, sondern immer gleiche textuelle Ordnungsstrukturen mit unterschiedlicher Funktion, bei deren Elementen äußere $\mathrm{Zu}$ griffspfade enden.

(c) Ein Wörterverzeichnis ist keine Zugriffsstruktur. Vielmehr weist ein Wörterverzeichnis in einem alphabetischen Wörterbuch eine alphabetische makrostrukturelle äußere Zugriffsstruktur auf. Dies heißt — anders ausgedrückt: Das Wörterverzeichnis ist der direkte Zugriffsbereich der alphabetischen makrostrukturellen äußeren Zugriffsstruktur und ist eine Menge von akzessiven Einträgen, die in diesem Fall Wörterbuchartikel sind.

Auch Registerzugriffsstrukturen sind äußere Zugriffsstrukturen. Diesen gilt unser nächster Einblick.

\subsection{Dritter Einblick: Einige Typen von Registerzugriffsstrukturen}

Derzeit existiert m.W. weder eine ausgearbeitete Theorie zu Registern, noch gibt es ein ausgearbeitetes typologisches System zu lexikographischen Registern (oder: Wörterbuchregistern). ${ }^{7}$ Entsprechend ist auch die Terminologie für die recht zahlreichen Typen von Wörterbuchregistern - wenigstens im Deutschen - derzeit noch unterentwickelt. Im Folgenden werden daher lediglich anhand von einigen Registern, die zu verschiedenen Registertypen gehören, 
grundlegende Eigenschaften von Registerzugriffsstrukturen erläutert, was allerdings nur möglich ist, wenn man auch einige wichtige Eigenschaften von Wörterbuchregistern berücksichtigt. Am Ende dieses Einblickes werden wir dann über die Grundbausteine einer Registertheorie verfügen.

Register können im Vorspann oder im Nachspann eines Printwörterbuches stehen; meistens stehen sie im Nachspann, bei Lieferungswerken und Mehrbändern ausschließlich dort. Jedes Register weist mindestens eine äußere Registerzugriffsstruktur auf, die auch registerinterne Zugriffsstruktur heißt (vgl. z.B. Wiegand 1989: 393f). Jedes Register ist eine geordnete Menge von Registereinträgen. Jeder Registereintrag ist ein akzessiver Wörterbucheintrag, der zu den direkt akzessiven Wörterbucheinträgen gehört (vgl. Abb. 1); er besteht aus einem Registereingang, der ein Element (der Trägermenge) der äußeren Registerzugriffsstruktur ist, sowie aus mindestens einer Registerangabe, die an den Registereingang adressiert ist. Ein Registereintrag heißt einfacher Registereintrag, wenn er genau eine solche Registerangabe aufweist, die direkt auf den Registereingang folgt; er heißt dagegen erweiterter Registereintrag, wenn er mehr als eine Registerangabe aufweist, die auf den Registereingang folgen. Hat er $\mathrm{n}$ Registerangaben, liegt ein $\mathrm{n}$-fach erweiterter Registereintrag vor. Der Registereintrag "ramponieren ital $1788 \mathrm{~V}^{\text {“ }}$ aus dem Alphabetischen Register des DFW ist z.B. ein dreifach erweiterter Registereintrag, weil drei Registerangaben auf „ramponieren“, den Registereingang, folgen. Alle drei Registerangaben sind an den Registereingang adressiert. Wird mit dem Registereingang ein sprachlicher Ausdruck genannt, ist der Registereingang stets auch eine Zeichengestaltangabe; diese zählt zu den Registerangaben genau so wie die Lemmazeichengestaltangabe zu den Artikelangaben gehört. Register, deren Registereinträge nur aus Registereingängen bestehen, die Zeichengestaltangaben sind, heißen reduzierte Register und weisen reduzierte Registereinträge auf. Die Menge aller derjenigen Registerangaben eines Registers, die auf einen Registereingang folgen, bildet den direkten Zugriffsbereich einer Registerzugriffsstruktur.

Wir betrachten zunächst Register, die Komponenten der Mediostruktur eines Wörterbuchs sind. Solche Register liegen vor genau dann, wenn alle Registerangaben, die auf Registereingänge folgen, nur Fundortdaten für die Ausführung von Verweisbefolgungshandlungen liefern, so dass alle diese Registerangaben als reduzierte Verweisangaben zu gelten haben, anhand derer der Benutzer Verweisadressen erschließen kann, die ihm als mediostrukturelle Leitelemente dienen. Solche Register weisen mediostrukturelle äußere Zugriffsstrukturen auf, die zu dem Untertyp der adressenvermittelnden Registerzugriffsstruktur gehören.

Eine adressenvermittelnde Registerzugriffsstruktur findet sich in Prosinger (1984). Da dieses Wörterbuch der alternativen Lexikographie auch weitere interessante Aspekte der Wörterbuchform aufweist, dient es im Folgenden als Beispielwörterbuch. Im vorliegenden Zusammenhang wichtige Aspekte seiner Wörterbuchform sind in Abb. 9 wiedergegeben. 


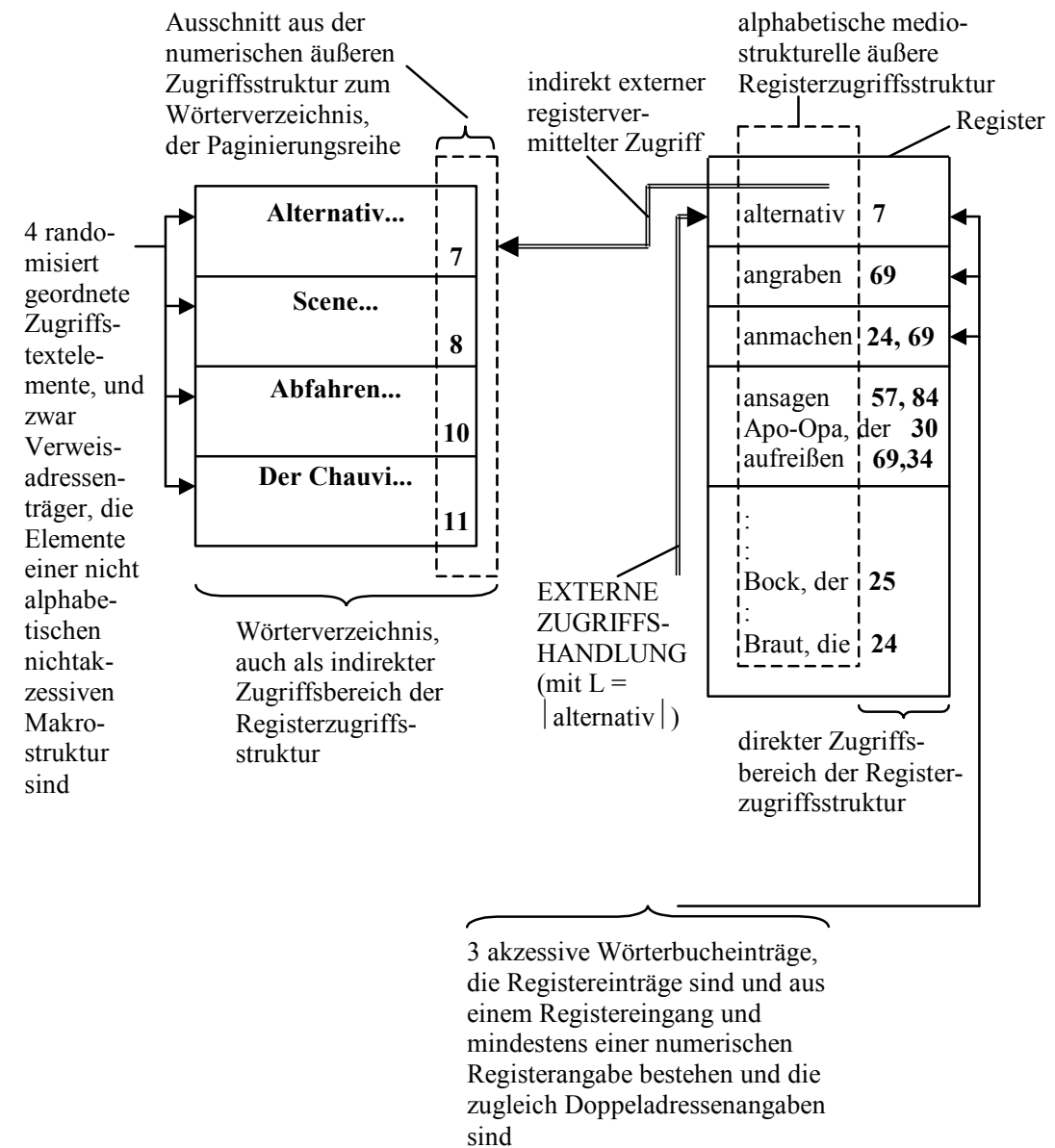

Abb. 9: Veranschaulichung zu einer alphabetischen mediostrukturellen äußeren Registerzugriffsstruktur

In Prosinger (1984) weist die Makrostruktur weder eine der alphabetischen noch eine der systematischen Anordnungsformen auf. Vielmehr sind die äußeren Zugriffstextelemente zufällig geordnet. Das bedeutet: Aus der Menge aller möglichen Reihenfolgen, die zu einer Menge von sprachlichen Ausdrücken gebildet werden können, ist eine Reihenfolge zufällig ausgewählt. Auf eine solche Makrostruktur kann nicht direkt extern zugegriffen werden. Zwar liefert z.B. das Wort Scene dem Benutzer die Buchstabenfolge |Scene $\mid$. Aber diese funktioniert nicht als Leitelement für den direkten externen Zugriff auf die Makrostruktur, da deren Elemente keine Leitelementträger und damit keine Lemmata sind, sondern lediglich Wörterbuchartikeltitel (kurz: Artikeltitel). Der Makrostruktur von Prosinger (1984) ist mithin keine makrostrukturelle Zugriffsstruktur inhärent. Betrachtet man diese Makrostruktur für sich alleine, liegt 
eine nichtakzessive Makrostruktur vom Typ der randomisierten Makrostruktur vor (vgl. auch Wiegand (2002: 419 u. 2003: 189ff). Prosinger (1984) wäre kein Wörterbuch ohne das Register im Nachspann, das in der Abb. 9 rechts ausschnittsweise abgebildet ist. Dieses Register weist eine alphabetische mediostrukturelle äußere Registerzugriffsstruktur auf, so dass man direkt extern auf dieses Register zugreifen kann. Entsprechend sind alle Registereingänge Leitelementträger. Im direkten Zugriffsbereich der Registerzugriffsstruktur finden sich nur Seitenangaben und damit nur Fundortangaben für denjenigen sprachlichen Ausdruck, der mit dem Registereingang genannt wird. Aus diesem Grund ist die Registerzugriffsstruktur eine adressenvermittelnde Zugriffsstruktur. Denn mit den numerischen Registerangaben, die auf den Registereingang folgen, werden numerische Verweisadressen genannt, die in der Paginierungsreihe von Prosinger (1984) liegen. Entsprechend ist die eleganteste Interpreta-

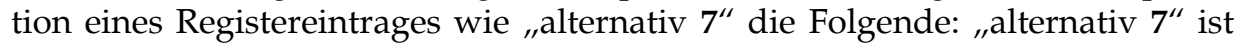
eine reduzierte Registerverweisangabe (also eine Verweisangabe, die als Teilangabe keine Verweisbeziehungsangabe, wie z.B. $\rightarrow$ aufweist). Mit ihr wird eine Verweisdoppeladresse genannt, die aus einer numerischen Verweishauptadresse, nämlich der Seitenzahl 7 besteht, sowie aus einer Verweisunteradresse, nämlich dem erwähnten Artikeltitel alternativ. Ein Benutzer, der mit einer Frage nach den Fundortdaten von alternativ im Zuge der Ausführung einer externen Zugriffshandlung den Registereintrag ,,alternativ 7“ erreicht hat, muss anhand dieses Eintrages einen Verweis erschließen und daraufhin eine externe Verweisbefolgungshandlung ausführen, die ihn zunächst zur Seitenzahl 7 und damit zur numerischen Hauptadresse $|7|$ führt, und danach auf der Wörterbuchseite 7 zum Artikelteil alternativ. Dieser fungiert damit als Träger der Verweisunteradresse |alternativ|. Alle Elemente der Makrostruktur in Prosinger (1984) sind damit Verweisadressenträger. Betrachtet man also die Makrostruktur in Prosinger (1984) nicht isoliert, sondern berücksichtigt die adressenvermittelnde Registerzugriffsstruktur, dann ist der Makrostruktur eine indirekt akzessive makrostrukturelle äußere Zugriffsstruktur inhärent.

An dieser Stelle sei zum präzisen Verständnis einer externen Zugriffshandlung, die entweder im unmittelbaren Ausführungsbereich einer Nachschlagehandlung oder im unmittelbaren Ausführungsbereich einer Verweisbefolgungshandlung liegt, Folgendes festgestellt: Der Zugriff, mit dem die Ausführung der jeweiligen Benutzungshandlung beendet ist, ist immer ein direkter Zugriff. Das Attribut indirekt in entsprechenden Mehrworttermini, die z.B. die verschiedenen Untertypen von akzessiven Wörterbucheinträgen bezeichnen, ist daher wie folgt zu verstehen: Der Benutzer kommt nur über eine andere zugriffsverschiedene (wörterbuchinterne) Position, in der er Fundortdaten (oder: neue Zugriffsdaten) als mediostrukturelle Leitelemente findet und damit in diesem Sinne „indirekt“ zum Benutzerziel, obwohl er auch mit der externen Zugriffshandlung, die im unmittelbaren Ausführungsbereich einer Verweisbefolgungshandlung liegt, ,"direkt" zugreift! Man kann die indirekte externe Datenakzessivität, um die es hier geht, auch wie folgt erläutern: Akzessive Wörter- 
bucheinträge und damit die Daten, die in ihnen textuell präsentiert werden, sind indirekt extern akzessiv genau dann, wenn die äußeren Zugriffstextelemente stets das Pfadende eines zusammengesetzten äußeren Zugriffspfades bilden. Der zusammengesetzte äußere Zugriffspfad besteht aus einem adkurrenten äußeren Zugriffspfad, an den sich unmittelbar ein binnenkurrenter äußerer Zugriffspfad anschließt.

Sucht beispielsweise ein Benutzer anhand des Leitelementes |alternativ | im Register von Prosinger (1984) den Registereingang ",alternativ“ und hat eine entsprechende externe Zugriffshandlung erfolgreich ausgeführt, dann wurde ein adkurrenter äußerer Zugriffspfad etabliert, dessen Pfadanfang das Leitelement |alternativ | und dessen Pfadende der Registereingang "alternativ" ist. Schließt sich dann direkt eine Verweisbefolgungshandlung an, die den Benutzer zum Wörterbuchartikel zu alternativ führt, wird ein binnenkurrenter äußerer Zugriffspfad atabliert, dessen Pfadende ein äußeres Zugriffstextelement, nämlich das Lemma alternativ ist. Betrachtet man die gesamte Wörterbuchbenutzung, dann wurde bis zur Erreichung des Benutzerziels ein zusammengesetzter äußerer Zugriffspfad etabliert. Der Weg zum Benutzerziel führt über das Register, so dass ein registervermittelter Zugriff erfolgt. In diesem Sinne sind die Wörterbuchartikel in Prosinger (1984) sowie die artikelinternen Daten nur indirekt extern akzessiv. Auch die Daten im tabellarischen Umtext des Wahrig (1989) sind nur indirekt extern akzessiv. Auf sie kann extern nur artikelvermittelt zugegriffen werden.

Im Folgenden betrachten wir anhand von Abb. 10 ein bifunktionales Äquivalentregister.

Bilingualisierte Fachwörterbücher, wie z.B. die geplanten WSK-Bände (vgl. zu diesen Schierholz und Wiegand 2005) oder polylingualisierte Fachwörterbücher, wie z.B. das Wörterbuch zur Lexikographie und Wörterbuchforschung (vgl. Wiegand 2003a sowie die Demoversion unter: www.fabulex.de), weisen häufig Äquivalentregister auf. In diesen werden z.B. — wie in der Abb. $10-$ die englischen Äquivalente mit den Registereingängen genannt, die in den Fachwörterbuchartikeln ebenfalls genannt und an die Lemmazeichengestaltangabe adressiert sind. Mit den Registerangaben von Äquivalentregistern werden die fachsprachlichen Lemmazeichen erwähnt. Möchte ein Benutzer z.B. wissen, wie das deutsche Äquivalent zu article constituent lautet, findet er die Antwort bereits im Äquivalentregister selbst: Das Äquivalent heißt Artikelkonstituente. Die Menge aller Registerangaben, die auf Registereingänge folgen, bilden daher den direkten Zugriffsbereich des Äquivalentregister. Eine Funktion des Äquivalentregisters besteht mithin darin, Benutzerfragen zu beantworten, die zum Fragentyp gehören: WIE HEISST DAS DEUTSCHE ÄQUIVALENT ZU EINEM ENGLISCHEN FACHAUSDRUCK X? Eine weitere Funktion des Äquivalentregisters besteht darin, dem Benutzer die Fundortdaten, also die lemmatischen Verweisaußenadressen als mediostrukturelle Leitelemente für die Ausführung von externen Verweisbefolgungshandlungen zu liefern. Demgemäß sind die Registerangaben zugleich reduzierte Registerverweisangaben, anhand 
derer artikelorientierte Verweise erschlossen werden können. Entsprechend ist das Wörterverzeichnis der indirekte Zugriffsbereich der alphabetischen Registerzugriffsstruktur, das mithin auch eine mediostrukturelle Funktion aufweist. Für Fachwörterbücher, die ein Äquivalentregister aufweisen, gilt, dass ihre Wörterbuchartikel zu den direkt und indirekt akzessiven Wörterbuchartikeln gehören. Man kann direkt extern auf sie zugreifen und registervermittelt. Anders ausgedrückt heißt das: Alle Lemmata können sowohl als Pfadende eines adkurrenten äußeren Zugriffspfades als auch als Pfadende eines zusammengesetzten äußeren Zugriffspfades auftreten.

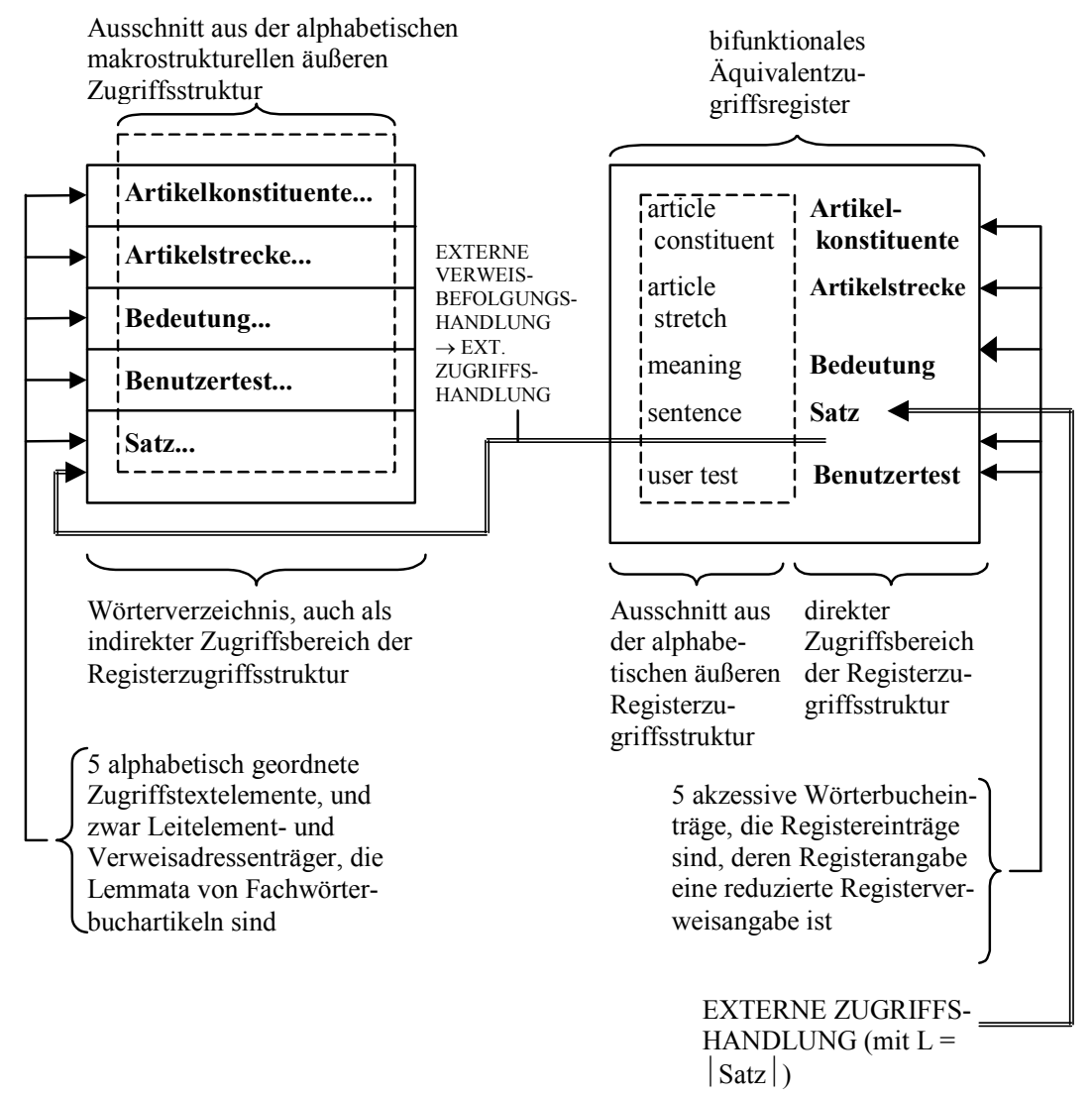

Abb. 10: Veranschaulichung zu einem bifunktionalen Äquivalentregister

Mit Blick auf die externe Datenakzessivität lässt sich auch eine Wörterbuchtypologie angeben. Wörterbücher mit Äquivalentregistern sind z.B. polyakzessiv, was bedeutet: $\mathrm{Zu}$ den makrostrukturellen äußeren Zugriffstextelementen gibt es stets zwei Zugriffspfade, die zu verschiedenen Typen gehören. Den polyakzessiven stehen die monoakzessiven Wörterbücher gegenüber, bei denen dies nicht der Fall ist. Sowohl der Typ des poly- als auch der des monoakzes- 
siven Wörterbuchs weisen mehrere Untertypen auf, worauf hier jedoch nicht näher eingegangen werden kann.

Im Folgenden betrachten wir anhand der Abb. 11 und 12 das Chronologische Register aus dem 7. Bd. des Deutschen Fremdwörterbuchs (Kirkness 1988).

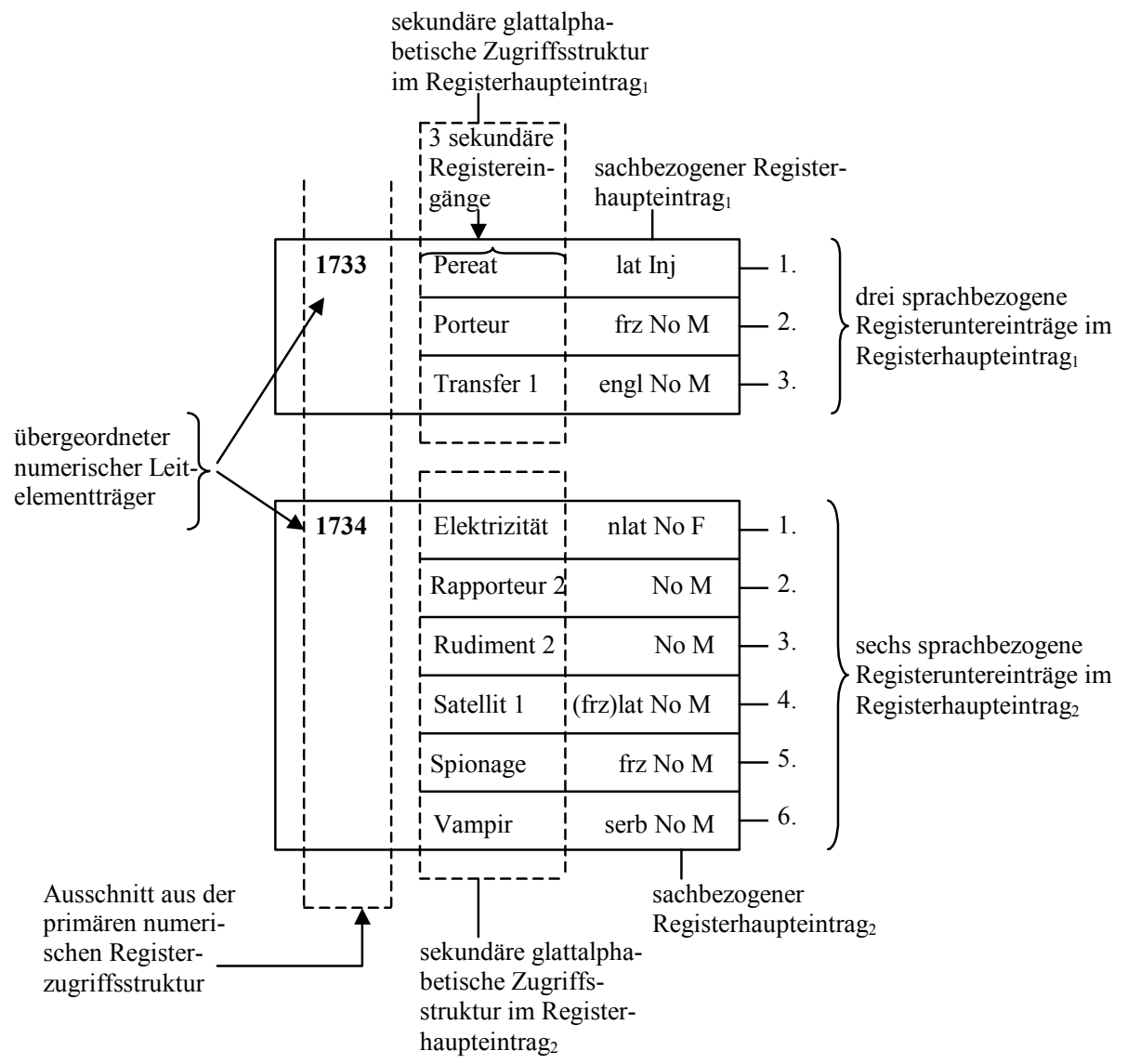

Abb. 11: Erste Veranschaulichung zum Chronologischen Register aus Kirkness (1988); vgl. auch Wiegand (2003: 186)

Das Chronologische Register in Kirkness (1988) gehört zu den Wörterbuchregistern, in denen zwei Zugriffsstrukturen gegeben sind. Zum Status der Zugriffsstrukturen sind zwei lexikographietheoretisch gut begründbare Positionen möglich. Nach der ersten Position liegen nur äußere Zugriffsstrukturen vor. Nach der zweiten Position ist die numerische Zugriffsstruktur eine äußere Zugriffsstruktur, und die glattalphabetischen Zugriffsstrukturen sind innere Zugriffsstrukturen von Registereinträgen. Ich stelle zunächst die erste theoretische Position anhand der Abb. 11 dar. 


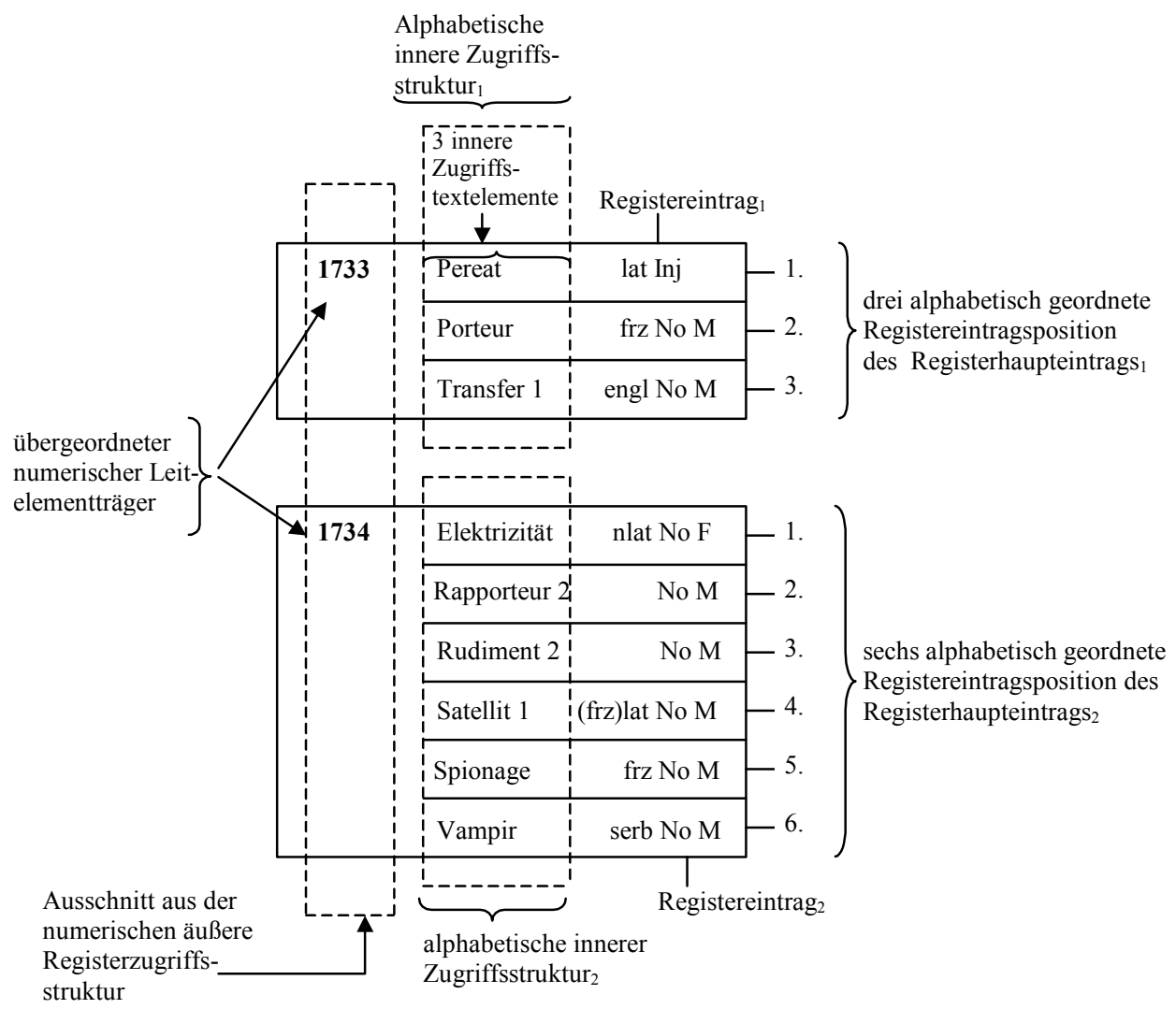

Abb. 12: Zweite Veranschaulichung zum Chronologischen Register aus Kirkness (1988)

Die primäre (oder: vorgeordnete) Zugriffsstruktur ist eine numerische äußere Registerzugriffsstruktur. Die sekundären (oder: nachgeordneten) Zugriffsstrukturen sind kleinere glattalphabetische äußere Registerzugriffsstrukturen. Die Leitelementträger der primären Registerzugriffsstruktur sind Jahreszahlen. Auf der Menge aller berücksichtigten Jahreszahlen ist eine zweistellige Ordnungsrelation definiert, auf deren Elemente (also geordnete Paare, deren erste Komponente z.B. 1733 und deren zweite Komponente 1734 ist) der Relationsterm $x$ geht $y$ zeitlich voraus zutrifft (mit ", $\mathrm{x}^{\prime \prime}$ und " $\mathrm{y}^{\prime \prime}$ als Variablen für Jahreszahlen). Die primäre Registerzugriffsstruktur ist damit eine lineare Ordnungsstruktur, auf die Benutzer z.B. mit Fragen vom Typ WELCHE WÖRTER WURDEN IM JAHR 1734 ENTLEHNT? direkt extern zugreifen können.

$\mathrm{Zu}$ jedem numerischen Leitelementträger als primärem Registereingang (oder: Registerhaupteingang) gehört ein Registerhaupteintrag. Jeder Registerhaupteintrag weist eine eigene sekundäre (oder: nachgeordnete) glattalphabetische äußere 
Zugriffsstruktur auf, deren Zugriffstextelemente die sekundären Registereingänge sind. Entsprechend ist z.B. 〈Pereat, Porteur, Transfer〉 die sekundäre glattalphabetische Zugriffsstruktur, die zum Registerhaupteintrag gehört (vgl. Abb. 11). Ein Registerhaupteintrag besteht aus mehreren Registeruntereinträgen, die mit sekundären Registereingängen beginnen. „Pereat lat Inj” (vgl. Abb. 11) ist dann ein Registeruntereintrag. Ein Benutzer, der den numerischen Registerhaupteingang (z.B. 1733) gefunden hat, kann daraufhin extern z.B. auf den sekundären Registereingang „Pereat" zugreifen.

Anhand der Abb. 12 wird im Folgenden die zweite theoretische Position erläutert. Nur die numerische Zugriffsstruktur wird als äußere Zugriffsstruktur angesehen. Es wird nicht zwischen Registerhaupt- und Registeruntereinträgen unterschieden; vielmehr weist ein Registereintrag n Registereintragspositionen auf (mit $n \geq 1$ ). Der Registereintrag 2 weist beispielsweise sechs Registereintragspositionen auf (vgl. Abb. 12). Die Registereintragspositionen sind nach ihrer ersten Angabe, der Fremdwortangabe, alphabetisch geordnet; dadurch ist eine alphabetische innere Zugriffsstruktur gegeben. Da auf der Menge aller Fremdwortangaben aller Registereinträge eine oberhalb-Relation definiert ist, sind alle inneren Zugriffsstrukturen aller Registereinträge des Chronologischen Registers in Kirkness (1988) vertikalarchitektonisch ausgebaut und damit glattalphabetische innere Zugriffsstrukturen.

Welche der beiden erläuterten theoretischen Positionen als die angemessenere zu gelten hat, kann nur in einem größeren theoretischen Rahmen entschieden werden, der in diesem Beitrag nicht zur Verfügung steht.

Obwohl es zahlreiche weitere Typen von Wörterbuchregistern gibt, kann der dritte Einblick hier abgebrochen werden: Die wichtigsten lexikographietheoretischen Begriffe, die benötigt werden, um Wörterbuchregister hinsichtlich ihrer Form und Funktion angemessen und exakt analysieren und anlegen zu können, wurden eingeführt. Für die Darstellung zahlreicher theoretischer Details sowie für die Entwicklung eines umfassenden Systems von Registertypologien wird wesentlich mehr Raum benötigt.

\section{Interne Datenakzessivität}

Wir wissen bereits aus dem 2. Abschnitt das Folgende: Die interne Datenakzessivität wird durch innere Zugriffsstrukturen hergestellt. Innere Zugriffsstrukturen findet man nur innerhalb von akzessiven Wörterbucheinträgen. Während für ein Printwörterbuch die externe Datenakzessivität eine conditio sine qua non ist, so dass mithin mindestens eine äußere Zugriffsstruktur für ein Printwörterbuch obligatorisch ist, ist die interne Datenakzessivität und damit auch das Vorhandensein von inneren Zugriffsstrukturen fakultativ. Beispielsweise weisen Wörterbücher, die nur nichtkondensierte Wörterbuchartikel (sensu Wiegand 2003: 207ff) aufweisen, die nur aus einem Lemma und einem Lemmakotext bestehen, niemals innere Zugriffsstrukturen auf. In solchen Artikeln kann man nur lesen, aber nicht — nachdem man das Lemma gefunden hat 
— durch die Ausführung von internen Zugriffshandlungen auf eine bestimmte textuelle Artikelposition gezielt zugreifen.

\subsection{Vierter Einblick: Einige Typen von inneren Zugriffsstrukturen}

Die wichtigste Unterscheidung bei den inneren Zugriffsstrukturen kann wie folgt angegeben werden: Es gibt innere Zugriffsstrukturen, die genau so wie äußere Zugriffsstrukturen funktionieren, und es gibt solche, bei denen das nicht der Fall ist. Die Erstgenannten sind die alphabetischen inneren Zugriffsstrukturen. Ein Beispiel sind die inneren Zugriffsstrukturen in den Registereinträgen des Chronologischen Registers in Kirkness (1988), wenn man die zweite Interpretation wählt (vgl. 3.3).

In zahlreichen Fachwörterbüchern finden sich am Ende des Artikels alphabetisch geordnete Literaturangaben, die insgesamt eine textuelle Artikelposition bilden, so dass diese Literaturposition dann eine positionenspezifische alphabetische innere Zugriffsstruktur aufweist.

Den alphabetischen stehen die nichtalphabetischen inneren Zugriffsstrukturen gegenüber. Sie unterscheiden sich grundsätzlich von allen äußeren Zugriffsstrukturen, da es keine allgemein bekannte, wörterbuchexterne Ordnungsstruktur gibt, nach deren Ordnungsgesetzlichkeiten innere Zugriffsstrukturen angelegt sind, so dass ein Benutzer, der diese Ordnungsgesetzlichkeiten kennt, auf innere Zugriffsstrukturen zugreifen könnte. Vielmehr muss die Kenntnis innerer Zugriffsstrukturen, vor allem die von partiell und vollständig kondensierten Wörterbuchartikeln, auf deren Betrachtung wir uns im Folgenden beschränken, durch die Lektüre entsprechender Ausschnitte der jeweils zuständigen Metatexte, z.B. durch die Lektüre der Benutzungshinweise, erst erworben werden. Dies bedeutet, dass nur der kundige Benutzer, also der, der die Metatexte gelesen hat und über entsprechende Kenntnisse bei der Wörterbuchbenutzung verfügt, interne Zugriffshandlungen ausführen kann.

Innere Zugriffsstrukturen werden in den Benutzungshinweisen dem Benutzer dadurch erklärt, dass ihm mehr oder weniger genau der so genannte Artikelaufbau oder das so genannte Grundschema für den Artikelaufbau erklärt werden. Untersucht man - und eine solche Untersuchung ist relativ schwierig und aufwendig -, was eigentlich in Wörterbüchern genau erklärt wird, wenn der "Artikelaufbau“ erklärt wird, dann ist das Ergebnis bei verschiedenen Wörterbüchern des gleichen Typs im Detail recht verschieden. Im Prinzip ist es aber gleichartig: Vor dem Hintergrund der lexikographietheoretisch bisher bekannten Strukturtypen von kondensierten Wörterbuchartikeln, nämlich dem Typ der Artikelkonstituentenstrukturen, dem Typ der Mikrostrukturen und dem Typ der Suchbereichsstrukturen wird unter dem Namen „Artikelaufbau“ (oder unter ähnlichen Namen) stets ein Mischtyp aus den drei genannten Typen erklärt. In den Erklärungen wird z.B. auf ausgewählte nichttypographische Strukturanzeigertypen (wie z.B. Kommata auf der Ebene der 
Wörterbuchform oder auf senkrechte Striche) und damit auf Elemente der Artikelkonstitutentenstruktur Bezug genommen. Zugleich wird auf bestimmte Typen von elementaren und nichtelementaren Angaben und auf Typen von charakteristischen Angabegruppen und damit sowohl auf Elemente der Artikelkonstituentenstruktur als auch auf solche der Mikrostruktur Bezug genommen. Weiterhin wird auf typographische Mikrostrukturanzeiger (wie z.B. kursiv und fett) hingewiesen und damit auf Eigenschaften von mikrostrukturellen Textkonstituenten; und schließlich werden auch ausgewählte Gestalteigenschaften der Artikeltexte berücksichtigt und damit Elemente von Suchbereichsstrukturen.

Von der in den Benutzungshinweisen erläuterten "Mischstruktur" behält der einzelne kundige Benutzer - wie ich anhand von Wörterbuchbenutzungsprotokollen zeigen konnte - meistens nur einen Strukturausschnitt. Bei verschiedenen Benutzern sind die kognitiv verfügbaren Strukturausschnitte verschieden. Dieses je individuelle Wissen von der in den Benutzungshinweisen erklärten Mischstruktur, mit der zugleich eine oder meistens mehrere innere Zugriffsstrukturen relativ zu bestimmten Artikeltypen beschrieben werden, fungiert als Orientierungs- und Zugriffshilfe für die Ausführung von inneren Zugriffshandlungen.

Während also die inneren Zugriffsstrukturtypen in den Metatexten als „Mischstrukturen" erklärt werden und in den einzelnen Wörterbuchartikeln der verschiedenen Typen als textuelle Mischstruktur tatsächlich auftreten und wahrgenommen werden können, fungiert als Zugriffshilfe für interne Zugriffshandlungen ein je individuelles Strukturwissen von den gegebenen Strukturen, das bei der Handlungsausführung korrigiert und ergänzt werden kann, so dass durch häufige Benutzung des gleichen Wörterbuchs aus dem kundigen allmählich ein erfahrener Benutzer (sensu Wiegand 1998: 508) wird.

Damit dürfte der grundsätzliche Unterschied zwischen alphabetischen äuBeren Zugriffsstrukturen und nichtalphabetischen inneren Zugriffsstrukturen unter dem Aspekt der Datenakzessivität klar sein: Alphabetische äußere Zugriffsstrukturen garantieren die externe Datenakzessivität jedem potentiellen Benutzer, der das Zugriffsalphabet beherrscht und gelernt hat, das alphabetische Sortieren nachzuvollziehen. Alle potentiellen Benutzer dieser Benutzerklasse sind sozusagen „vor den Gesetzen des Alphabets“ gleich. Jeder kann auf jedes äußere Zugriffstextelement punktgenau zugreifen. Der eine kann es schneller, der andere langsamer. Und keiner muss die alphabetische äußere Zugriffsstruktur in dem Sinne „im Kopf" haben, dass er die genaue Reihenfolge aller alphabetisch geordneten äußeren Zugriffstextelemente kennt.

Nichtalphabetische innere Zugriffsstrukturen muss ein Benutzer — wenn er interne Zugriffshandlungen ausführen möchte, die zu einem punktgenauen Zugriff führen - dagegen so „im Kopf " haben, wie sie in den Metatexten beschrieben sind, und das bedeutet: Er muss eine bestimmte Reihenfolge von unterschiedlichen Textsegmenttypen „im Kopf " haben und diese gegebenenfalls für unterschiedliche Artikeltypen variieren können. 


\subsection{Fünfter Einblick: Bemerkungen zu inneren Schnellzugriffsstrukturen}

Um die Eigenart innerer Schnellzugriffsstrukturen leichter erkennen zu können, ist es nützlich, kurz einen Blick auf die äußeren Schnellzugriffsstrukturen zu werfen, und zwar anhand der beiden wohl bekanntesten Typen. Manche alphabetische Wörterbücher weisen zur Erleichterung des direkten externen Zugreifens ein Daumenregister auf. Dieses ist der Träger einer alphabetischen äußeren Schnellzugriffsstruktur 〈A, B, C, ..., Z〉. Die äußeren Zugriffsstrukturanzeiger A, B, C, ..., Z, die im Wörterverzeichnis vor den Artikelstrecken stehen, werden auf den Ausschnitten des Registers erwähnt, so dass der Anfang einer Artikelstrecke leichter gefunden werden kann. Ein Daumenregister kann wesentlich dazu beitragen, dass die äußeren Zugriffszeiten kürzer sind.

Das zweite Beispiel ist eine alphabetische lebende Lemmakolumne. Meistens ist sie so gestaltet, dass auf zwei aufgeschlagenen Wörterbuchseiten auf der linken Seite über dem Wörterverzeichnis das erste Lemma dieser Seite genannt wird und auf der rechten Seite das letzte Lemma dieser Seite. Der Benutzer kann dann schneller entscheiden, ob das gesuchte Lemma auf den beiden aufgeschlagenen Seiten zu finden ist oder nicht.

Um die Vorteile, die eine alphabetische äußere Zugriffsstruktur bietet, nutzen zu können, muss der Benutzer nur das Alphabet kennen. Dies ist bei nichtalphabetischen inneren Schnellzugriffsstrukturen anders. Diese sind Teilstrukturen innerer Zugriffsstrukturen. Zu ihrer Trägermenge gehören nur besonders markante strukturanzeigende Angaben und nichttypographische Strukturanzeiger. Diese, ihre Reihenfolge sowie die wesentlichen Angabetypen (zu der die Angaben gehören, die auf sie folgen) muss der Benutzer kennen. Dies sei an einem einfachen Beispiel erläutert.

Riemen, der; -s, - 1. Streifen aus Leder, festem Gewebe, Gummi, synthetischem Material: ein langer, schmaler R.; einen R. verkürzen, zurechtschneiden, um etw. schnallen; die Hose wird mit einem R. (Gürtel) gehalten - 2. endloses Band aus Leder, Gummi, festem Gewebe, mit dessen Hilfe Drehmomente von einer Welle auf eine andere weitergeleitet werden - 3. Rudern zur Fortbewegung eines Ruderbootes (paarweise) verwendete, an einem Ende $z$ u einem Blatt verbreiterte hölzerne Stange: die R. einziehen, ergreifen; sich in die R. legen (kräftig rudern)

+ umg. den R. enger schnallen (sich einschränken); salopp sich am $\mathrm{R}$. reißen (sich anstrengen, sich zusammennehmen)

Abb. 13: Wörterbuchartikel wa 1 aus HWDG 
Die innere Schnellzugriffsstruktur von wa ${ }_{1}$ kann wie folgt angegeben werden: $\langle$ Riemen $<1 .<2 .<3 .<+>$.

Ein kundiger Benutzer, der mit einer Frage „Welche Phraseme mit Riemen sind gebucht?" eine externe Zugriffshandlung erfolgreich ausgeführt und damit das Lemma Riemen erreicht hat, kann sofort auf die Phrasemidentifizierungsangabe intern zugreifen, die durch ", ${ }^{\prime \prime}$ realisiert wird, weil er weiß, dass die Phrasemangaben am Schluss des Artikels nach "," also nicht den ganzen Artikel durchsuchen. Dadurch verkürzt sich die innere Zugriffszeit deutlich. Die innere Schnellzugriffsstruktur in wa ${ }_{1}$ gehört zu den nicht glatten inneren Schnellzugriffsstrukturen; bei glatten inneren Schnellzugriffsstrukturen ist auf den Elementen der Trägermenge neben einer Präzedenzrelation noch eine oberhalb-Relation definiert. Dies ist in wa ${ }_{1}$ nicht der Fall.

\section{Schlussbemerkung}

In diesem einführenden Beitrag wurde versucht, die wichtigsten Eigenschaften der Wörterbuchform, die mit der Herstellung der Datenakzessivität in Printwörterbüchern zusammenhängen, herauszuarbeiten. Die Vielfalt der Ausprägungen dieser Eigenschaften sowie die Fülle ihrer unterschiedlichen Kombinationsmöglichkeiten konnten dabei nur angedeutet werden. Die zentralen Begrifflichkeiten, um das Datenakzessivitätsprofil von Printwörterbüchern genau beschreiben zu können, wurden jedoch bereit gestellt.

\section{Anmerkungen}

1. Ein für die Wörterbuchforschung angemessener Wissensbegriff sowie eine passende Terminologie werden in Wiegand (2002) und (2002a) vorgeschlagen.

2. Integrierte Umtexte sind solche Umtexte, in denen Daten zu finden sind, die dazu beitragen, dass ein Wörterbuch seinen genuinen Zweck erfüllt; meistens sind integrierte Umtexte mit dem Wörterverzeichnis mediostrukturell vernetzt.

3. Akzessive Daten finden sich nur in akzessiven Wörterbucheinträgen; oder anders ausgedrückt: Akzessive Daten sind nur solche Daten, auf die anhand mindestens einer äußeren Zugriffsstruktur extern zugegriffen werden kann.

4. Solche Wörterbücher finden sich z.B. bei den frühen deutschen Rechtschreibwörterbüchern. Alphabetische Mehrbänder weisen eine vorgelagerte alphabetische Zugriffsstruktur auf. Diese findet sich auf dem Buchrücken und hat bei einem Zweibänder z.B. die Form A-K, L-Z.

5. Vgl. z.B. Günther 1996.

6. Für spezifischere Zusammenhänge lassen sich Suchpfade von Zugriffspfaden unterscheiden. Zugriffspfade sind dann solche Suchpfade, die von erfolgreichen Zugriffshandlungen etabliert werden, also solchen, die als Ergebnis einen Zugriff aufweisen.

7. Kirkness (1989) ist lediglich eine nützliche Beschreibung einiger Funktionen von Registern und eine Sammlung von Beispielen, wo sich Register mit bestimmten Strukturen und Funktionen finden lassen. 


\section{Literatur}

Bergenholtz, H. und S. Tarp (Hrsg.). 1995. Manual of Specialised Lexicography. Amsterdam/Philadelphia: John Benjamins.

Bergenholtz, Henning, Sven Tarp und Herbert Ernst Wiegand. 1999. Datendistributionsstrukturen, Makro- und Mikrostrukturen in neueren Fachwörterbüchern. Hoffmann, L., H. Kalverkämper und H.E. Wiegand (Hrsg.). 1998-1999. Fachsprachen. Ein internationales Handbuch zur Fachsprachenforschung und Terminologiewissenschaft/Languages for Special Purposes. An International Handbook of Special-Language and Terminology Research: 1762-1832. Berlin/New York: Walter de Gruyter.

DFW = Kirkness, Alan (Hrsg.). 1988. Deutsches Fremdwörterbuch. Begonnen von Hans Schulz, fortgeführt von Otto Basler, weitergeführt im Institut für deutsche Sprache. 7. Bd. Berlin/New York: Walter de Gruyter.

Duden-2GW = Duden. 1993-1995. Das große Wörterbuch der deutschen Sprache in acht Bänden. 2. völlig neu bearb. u. stark erw. Aufl. Hrsg. u. bearb. v. Wissenschaftlichen Rat u. den Mitarbeitern der Dudenredaktion unter der Leitung v. Günther Drosdowski. Mannheim: Dudenverlag.

Günther, Hartmut. 1996. Schrift als Zahlen- und Ordnungssystem - alphabetisches Sortieren. Günther, H. und O. Ludwig (Hrsg.). 1994-1996. Schrift und Schriftlichkeit. Ein interdisziplinäres Handbuch internationaler Forschung/Writing and Its Use. An Interdisciplinary Handbook of International Research: 1568-1583. Berlin/New York: Walter de Gruyter.

Hausmann, Franz Josef und Herbert Ernst Wiegand. 1989. Component Parts and Structures of General Monolingual Dictionaries: A Survey. Hausmann, F.J., O. Reichmann, H.E. Wiegand und L. Zgusta (Hrsg.). 1989-1991: 329-360.

Hausmann, F.J., O. Reichmann, H.E. Wiegand und L. Zgusta (Hrsg.). 1989-1991. Wörterbücher. Ein internationales Handbuch zur Lexikographie/Dictionaries. An International Encyclopaedia of Lexicography/Dictionnaires. Encyclopédie internationale de lexicographie. Berlin/New York: Walter de Gruyter.

HWDG = Kempcke, Günter et al. (Hrsg.). 1984. Handwörterbuch der deutschen Gegenwartssprache. In zwei Bänden. Berlin: Akademie-Verlag.

Kirkness, Alan (Hrsg.). 1988. Deutsches Fremdwörterbuch. Begonnen v. Hans Schulz, fortgeführt v. Otto Basler, weitergeführt im Institut für deutsche Sprache. 7. Bd. Berlin/New York: Walter de Gruyter.

Kirkness, Alan. 1989. Wörterbuchregister. Hausmann, F.J., O. Reichmann, H.E. Wiegand und L. Zgusta (Hrsg.). 1989-1991: 767-771.

Mackensen, Lutz. 1977. Deutsches Wörterbuch. Rechtschreibung. Grammatik. Stil. Worterklärung. Fremdwörterbuch. Geschichte des deutschen Wortschatzes. 9. völlig neubearb. u. stark erw. Aufl. München: Südwest Verlag.

Prosinger, Wolfgang. 1984. Das rabenstarke Lexikon der Scene-Sprache. Der große Durchblick für alle Freaks, Spontis, Schlaffis, Softies, Flipper und Hänger sowie deren Verwandte und sonstige Fuzzis. Illustriert von Peter Gaymann. Frankfurt am Main: Eichborn Verlag.

Schierholz, Stefan und Herbert Ernst Wiegand. 2005. Die Wörterbücher zur Sprach- und Kommunikationswissenschaft. Eine neue Konzeption der linguistischen Fachlexikographie und ihre computergestützte Praxis. Lexicographica 20: 164-264.

Steyn, Mariza. 2004. The Access Structure in Learner's Dictionaries. Lexikos 14: 275-298. 
Wahrig, Gerhard (Hrsg.). 1989. dtv-Wörterbuch der deutschen Sprache. 10. Aufl. München: Deutscher Taschenbuchverlag.

Wiegand, Herbert Ernst. 1989. Aspekte der Makrostruktur im allgemeinen einsprachigen Wörterbuch: Alphabetische Anordnungsformen und ihre Probleme. Hausmann, F.J., O. Reichmann, H.E. Wiegand und L. Zgusta (Hrsg.). 1989-1991: 246-280.

Wiegand, Herbert Ernst. 1998. Wörterbuchforschung. Untersuchungen zur Wörterbuchbenutzung, zur Theorie, Geschichte, Kritik und Automatisierung der Lexikographie. 1. Teilband. Mit 159 Illustrationen im Text. Berlin/New York: Walter de Gruyter.

Wiegand, Herbert Ernst. 1998a. Altes und Neues zur Makrostruktur in alphabetischen Printwörterbüchern. Wiegand, H.E. (Hrsg.). Wörterbücher in der Diskussion III. Vorträge aus dem Heidelberger Lexikographischen Kolloquium: 348-372. Lexicographica. Series Maior 84. Tübingen: Max Niemeyer.

Wiegand, Herbert Ernst. 2002. Zur Makrostruktur und zu den äußeren Zugriffsstrukturen im de Gruyter Wörterbuch Deutsch als Fremdsprache. Wiegand, Herbert Ernst (Hrsg.). 2002. Perspektiven der pädagogischen Lexikographie des Deutschen II. Untersuchungen anhand des de Gruyter Wörterbuch Deutsch als Fremdsprache: 413-442. Lexicographica. Series Maior 110. Tübingen: Max Niemeyer.

Wiegand, Herbert Ernst. 2002a. Altes und Neues zur Mediostruktur in Printwörterbüchern. Lexicographica 18: 168-252.

Wiegand, Herbert Ernst. 2003. Überlegungen zur Typologie von Wörterbuchartikeln in Printwörterbüchern. Ein Beitrag zur Theorie der Wörterbuchform. Lexicographica 19: 169-313.

Wiegand, Herbert Ernst. 2003a. Wörterbuch zur Lexikographie und Wörterbuchforschung/Dictionary of Lexicography and Dictionary Research. Städtler, Thomas (Hrsg.). 2003. Wissenschaftliche Lexikographie im deutschsprachigen Raum. Im Auftrag der Heidelberg Akademie der Wissenschaften: 417-437. Heidelberg: Winter Verlag. 